\title{
DEDUALIZING COMPLEXES OF BICOMODULES AND MGM DUALITY OVER COALGEBRAS
}

\author{
LEONID POSITSELSKI
}

\begin{abstract}
We present the definition of a dedualizing complex of bicomodules over a pair of cocoherent coassociative coalgebras $\mathcal{C}$ and $\mathcal{D}$. Given such a complex $\mathcal{B} \bullet$, we construct an equivalence between the (bounded or unbounded) conventional, as well as absolute, derived categories of the abelian categories of left comodules over $\mathcal{C}$ and left contramodules over $\mathcal{D}$. Furthermore, we spell out the definition of a dedualizing complex of bisemimodules over a pair of semialgebras, and construct the related equivalence between the conventional or absolute derived categories of the abelian categories of semimodules and semicontramodules. Artinian, co-Noetherian, and cocoherent coalgebras are discussed as a preliminary material.
\end{abstract}

\section{Contents}

1. Introduction 1

2. Coalgebras with Finiteness Conditions 9

3. MGM Duality for Coalgebras 19

4. MGM Duality for Semialgebras 26

References 33

\section{INTRODUCTION}

1.1. In the classical homological algebra, one was not supposed to consider unbounded derived categories; certainly not when working with categories or functors of infinite homological dimension. Right derived functors were acting from bounded below derived categories, while left derived functors were defined on bounded above derived categories. Such derived functors were constructed using resolutions by complexes of injective or projective objects. More generally, one would consider resolutions by complexes with the terms adjusted to the particular functor in question, such as flat modules or flasque sheaves.

Everything changed after the watershed paper of Spaltenstein [34], which explained, following the idea of Bernstein, how to work with unbounded complexes, particularly when constructing derived functors of infinite homological dimension. The key innovation was to strengthen the conditions imposed on resolutions and work with what came to be known as the homotopy injective or homotopy projective 
complexes. Unlike in the classical homological algebra, these are not termwise conditions: whether a complex is homotopy injective or homotopy projective depends on the differential in the complex and not only on its terms.

The unbounded derived category of modules over an associative ring turned out to be particularly well-behaved. In the subsequent work of Keller, Bernstein-Lunts, and Hinich [15, 2, 9], the theory was extended to DG-modules over DG-rings. The unbounded derived category of DG-modules $\mathrm{D}(A$-mod $)$ is compactly generated, and it only depends on the quasi-isomorphism class of a DG-ring $A$. One can generalize even further and replace an associative DG-ring $A$ with an $\mathrm{A}_{\infty}$-algebra.

The theory that grew out of Spaltenstein's paper became so hugely popular that nowadays people use the homotopy injective and homotopy projective resolutions even when they are not actually relevant. That is what was happening in the case of the MGM (Matlis-Greenlees-May) duality/equivalence theory [23]. In fact, in the MGM theory one deals with derived functors of finite homological dimension, and the use of complexes of adjusted objects, similar to that of (complexes of) flasque or soft sheaves in the computation of sheaf cohomology/derived direct images, is called for [29].

1.2. The next development, which came about a decade later, was that people started to work with complexes viewed up to equivalence relations more delicate than the conventional quasi-isomorphism $[10,18,16]$. In other words, triangulated categories in which some, though not too many, acyclic complexes survive as nonzero objects attracted a certain interest. In addition to the constructions of compact generators $[13,17]$, one of manifestations of the phenomenon which the present author calls the derived co-contra correspondence was first noticed in the paper [12].

The present author's own ideas about the subject were published with about a decade-long delay $[24,25]$. Developed originally in the context of derived nonhomogeneous Koszul duality, they proceed from the observation that replacing the conventional quasi-isomorphism of complexes with more delicate equivalence relations is a natural alternative to strengthening the conditions on resolutions when working with unbounded complexes. In the terminology going back to the classical paper [11] (where two kinds of differential derived functors were introduced), this point of view came to be known as the distinction between two kinds of derived categories.

In the derived categories of the first kind, complexes are considered up to the conventional quasi-isomorphism (which does not depend on the module structure on the complexes, but only on their underlying complexes of abelian groups), which necessitates the use of homotopy adjusted complexes as resolutions (meaning the conditions on resolving complexes depending on the differentials and not only on the underlying graded object structures). In the derived categories of the second kind, some acyclic complexes survive as nonzero objects (and the equivalence relation on complexes depends on their module structures and not only on the underlying complexes of abelian groups), while the conditions on resolutions do not depend on the differentials on them (but only on their underlying graded objects). 
Another advantage of derived categories of the second kind is that they are defined for curved differential graded (CDG) structures as well as for conventional differential graded structures [25]. Hence the important role that such derived category constructions play, in particular, in the theory of matrix factorizations $[22,5,1]$.

The conventional derived category is the derived category of the first kind. The two most important versions of derived categories of the second kind are the coderived and the contraderived category. In well-behaved situations, the coderived category of (curved) DG-modules is equivalent to the homotopy category of (curved) DG-modules whose underlying graded modules are injective, while the contraderived category of (curved) DG-modules is equivalent to the homotopy category of (curved) DG-modules whose underlying graded modules are projective.

1.3. It turned out that the conventional derived categories of DG-comodules over DG-coalgebras (over a field) are not as well-behaved as the derived categories of DG-modules. The derived category of DG-comodules over a DG-coalgebra can change when the DG-coalgebra is replaced by a quasi-isomorphic one [14], [25, Remark 2.4]. There are no obvious reasons why the derived category $\mathrm{D}(\mathcal{C}$-comod $)$ of $\mathrm{DG}$-comodules over a DG-coalgebra $\mathcal{C}$ (or even complexes of comodules over a coalgebra $\mathcal{C}$ ) should be compactly generated, though one can show that it is well-generated [25, Section 5.5].

Perhaps one is not supposed to consider the conventional derived category of complexes of comodules (or DG-comodules) at all. The notion that one should work with the derived categories of modules and the coderived categories of comodules goes back to $[18,16]$. The monograph [24] is based on the philosophy that one is supposed to take the derived category of modules, the coderived category of comodules, and the contraderived category of contramodules.

In fact, for any curved DG-coalgebra $\mathcal{C}$ over a field $k$, the coderived category $\mathrm{D}^{\mathrm{co}}(\mathcal{C}$-comod) of left curved $\mathrm{DG}$-comodules over $\mathcal{C}$ is compactly generated (by the bounded derived category of $k$-finite-dimensional CDG-comodules). The coderived category of CDG-comodules is also equivalent to the homotopy category of CDG-comodules with injective underlying graded comodules.

Furthermore, there is a natural equivalence between the coderived category of left CDG-comodules and the contraderived category of left CDG-contramodules over $\mathcal{C}$ [25, Section 5]:

$$
\mathrm{D}^{\mathrm{co}}(\mathcal{C}-\text { comod }) \simeq \mathrm{D}^{\mathrm{ctr}}(\mathcal{C}-\text { contra }) .
$$

The contraderived category of CDG-contramodules is equivalent to the homotopy category of CDG-contramodules with projective underlying graded contramodules. The triangulated equivalence (1) is a principal example of what is called the derived comodule-contramodule correspondence phenomenon in [24, 25].

1.4. Several words about the contramodules are due at this point. There are two abelian categories associated naturally with an associative ring $A$ : the left $A$-modules and the right $A$-modules. In contrast, for a coalgebra $\mathcal{C}$ (say, over a field $k$ ) there are four such abelian categories: the left and the right $\mathcal{C}$-comodules, and the left and the right $\mathcal{C}$-contramodules. The categories of comodules have exact functors of filtered 
inductive limit and enough injective objects. The categories of contramodules have exact functors of infinite product and enough projective objects.

Let $\mathcal{C}^{*}$ denote the dual $k$-vector space to $\mathcal{C}$, endowed with its natural structure of a pro-finite-dimensional topological $k$-algebra. Then the $\mathcal{C}$-comodules are the same thing as discrete $\mathrm{C}^{*}$-modules, while the $\mathcal{C}$-contramodules form an "intermediate" category between arbitrary $\mathcal{C}^{*}$-modules and pseudo-compact (pro-finite-dimensional) topological $\mathfrak{C}^{*}$-modules. The latter form a category equivalent to the opposite category to $\mathcal{C}$-comodules. More precisely, there are natural forgetful functors

$$
(\text { comod-C })^{\text {op }} \longrightarrow \mathcal{C} \text {-contra } \longrightarrow \mathrm{C}^{*}-\bmod
$$

from the opposite category to right $\mathcal{C}$-comodules to left $\mathcal{C}$-contramodules and to left $\mathcal{C}^{*}$-modules (in addition to the fully faithful functor $\mathcal{C}$-comod $\longrightarrow \mathcal{C}^{*}$-mod identifying left $\mathcal{C}$-comodules with discrete left $\mathcal{C}^{*}$-modules).

In other words, $\mathcal{C}$-contramodules can be viewed as a species of "complete" (as opposed to discrete) $\mathfrak{C}^{*}$-modules. Nevertheless, contramodules carry no underlying topologies on them. Instead, they are discrete $k$-vector spaces endowed with infinite summation operations with the coefficients in $\mathcal{C}^{*}$ [28].

1.5. As we have already mentioned, the coderived categories of comodules and the contraderived categories of contramodules are better behaved than the conventional (unbounded) derived categories of comodules or contramodules. In other words, considering derived categories of the first kind along the ring variables and derived categories of the second kind along the coalgebra variables produces the better behaved triangulated categories [24].

Still, there is something to be said about the conventional derived categories of DG-comodules and DG-contramodules, too. The following results can be found in [25, Theorem 2.4 and Section 5.5] (for part (d), one has to look into the postpublication arXiv version of [25]).

Theorem 1.1. Let $\mathcal{C}$ be a DG-coalgebra over a field $k$. Then

(a) the Verdier quotient functor $\mathrm{D}^{\mathrm{ctr}}(\mathcal{C}$-contra $) \longrightarrow \mathrm{D}(\mathcal{C}$-contra) has a (fully faithful) left adjoint functor $\mathrm{D}(\mathrm{C}$-contra $) \longrightarrow \mathrm{D}^{\mathrm{ctr}}(\mathrm{C}$-contra $)$;

(b) the essential image of the triangulated functor $\mathrm{D}(\mathrm{C}$-contra $) \longrightarrow \mathrm{D}^{\mathrm{ctr}}(\mathrm{C}$-contra $)$ is the minimal full triangulated subcategory in $\mathrm{D}^{\mathrm{ctr}}(\mathrm{C}$-contra) containing the left $D G$-contramodule $\operatorname{Hom}_{k}(\mathcal{C}, k)$ over $\mathcal{C}$ and closed under infinite direct sums;

(c) the Verdier quotient functor $\mathrm{D}^{\mathrm{co}}(\mathrm{C}$-comod $) \longrightarrow \mathrm{D}(\mathrm{C}$-comod) has a (fully faithful) right adjoint functor $\mathrm{D}(\mathrm{C}-$ comod $) \longrightarrow \mathrm{D}^{\mathrm{co}}(\mathrm{C}-$ comod $)$;

(d) assuming Vopénka's principle in set theory, the essential image of the triangulated functor $\mathrm{D}(\mathrm{C}-\mathrm{comod}) \longrightarrow \mathrm{D}^{\mathrm{co}}(\mathrm{C}-\mathrm{comod})$ is the minimal full triangulated subcategory in $\mathrm{D}^{\mathrm{co}}(\mathcal{C}$-comod) containing the left DG-comodule $\mathcal{C}$ over $\mathcal{C}$ and closed under infinite products.

It should be added that the triangulated equivalence (1) takes the DG-comodule $\mathcal{C}$ over $\mathcal{C}$ to the DG-contramodule $\operatorname{Hom}_{k}(\mathcal{C}, k)$ over $\mathcal{C}$. Thus, assuming Vopěnka's principle, the derived categories $\mathrm{D}(\mathrm{C}-$ comod $)$ and $\mathrm{D}(\mathcal{C}$-contra $)$ are related as two full 
triangulated subcategories in the same triangulated category (1), generated by the same object in this category; but one of them is generated using shifts, cones, and infinite products, while the other one is generated using shifts, cones, and infinite direct sums. Both the (set-indexed) direct sums and products of arbitrary objects exist in the compactly generated triangulated category (1) (but the object $\mathrm{C} \longleftrightarrow$ $\operatorname{Hom}_{k}(\mathcal{C}, k)$ is not compact in this category).

1.6. The aim of this paper is to demonstrate a set of (admittedly, rather restrictive) assumptions and additional data allowing to construct an equivalence between the derived category of complexes of comodules over a coalgebra $\mathcal{C}$ and the derived category of complexes of contramodules over another coalgebra $\mathcal{D}$,

$$
\mathrm{D}^{\star}(\mathcal{C} \text {-comod }) \simeq \mathrm{D}^{\star}(\mathcal{D} \text {-contra }) \text {. }
$$

Here the symbol $\star$ means that both the bounded and unbounded conventional derived categories are allowed, i. e., one can have $\star=b,+,-$, or $\varnothing$.

Moreover, the triangulated equivalence (2) also holds for the absolute derived categories with the symbols $\star=$ abs + , abs - , or abs, which are versions of the construction of derived categories of the second kind introducted in [25] and [27, Appendix A]. Unlike in (1), though, the derived category symbol must be the same in the left and the right-hand side of the equivalence (2).

The triangulated equivalence (2), connecting the conventional derived categories of comodules and contramodules, is a species of what can be called the "naive derived co-contra correspondence". In the present author's work, it first appeared in the algebro-geometric setting as an equivalence between the derived categories of quasicoherent sheaves and contraherent cosheaves over a quasi-compact semi-separated scheme [27, Section 4.6] (or alternatively, over a Noetherian scheme of finite Krull dimension [27, Theorem 5.8.1]). In the subsequent papers [29, 32], the same principle was applied in order to formulate the $M G M$ duality/equivalence and the triangulated Matlis equivalence.

1.7. The equivalence of categories (2) can be called the "MGM duality for coalgebras". A bit of history of the MGM duality is worth recalling in this connection. The three-letter abbreviation stands for Matlis-Greenlees-May [20, 7]. The related chain of results can be further traced to the seminal paper of Harrison [8], where certain equivalences of additive subcategories in the category of abelian groups were constructed. Matlis extended these to equivalences between additive subcategories in the category of modules over an arbitrary commutative domain [19].

In the paper [20], which came more than a decade later, Matlis constructs an equivalence between certain additive subcategories in the category of modules over a commutative ring $R$ related to an ideal $I \subset R$ generated by a regular sequence. Greenlees and May [7] initiated the study of the derived functors of $I$-adic completion for an arbitrary finitely generated ideal $I$ in a commutative ring $R$. Dwyer and Greenlees [4] formulated the theory in the form of a triangulated equivalence between two full subcategories (of what we would now call the " $I$-torsion" and " $I$-complete" complexes $)$ in the derived category $\mathrm{D}(R$-mod $)$ of modules over a commutative ring 
$R$ with a finitely generated ideal $I \subset R$. Porta, Shaul, and Yekutieli [23] studied the case of a weakly proregular finitely generated ideal $I$.

The present author's paper [29], which formulated the theory in its state-of-the-art form, emphasized and discussed the role of what it called a dedualizing complex of $I$-torsion $R$-modules in the MGM duality theory. It also demonstrated the central role of the abelian category of $I$-contramodule $R$-modules, on par with the much more familiar dual-analogous abelian category of $I$-torsion $R$-modules, in the MGM duality.

1.8. The main results of the MGM duality theory, as formulated in [29], are the following ones. Given a finitely generated ideal $I$ in a commutative ring $R$, denote by $R$ - $\bmod _{I \text {-tors }}$ and $R$-mod $I$-ctra $\subset R$-mod the abelian subcategories of $I$-torsion and $I$-contramodule $R$-modules. (See [31] for an introductory discussion of these subcategories.) Then for every conventional derived category symbol $\star=b,+,-$, or $\varnothing$ there is a natural triangulated equivalence

$$
\mathrm{D}_{I \text {-tors }}^{\star}(R-\text { mod }) \simeq \mathrm{D}_{I \text {-ctra }}^{\star}(R-\text { mod })
$$

between the full subcategory of complexes of $R$-modules with $I$-torsion cohomology modules and the full subcategory of complexes of $R$-modules with $I$-contramodule cohomology modules in $\mathrm{D}^{\star}(R-$ mod $)$.

Furthermore, assuming that the ideal $I \subset R$ is weakly proregular (which always holds, e. g., when the ring $R$ is Noetherian), for every derived category symbol $\star=\mathrm{b}$, ,,$+- \varnothing$, abs + , abs-, or abs, there is a natural equivalence between the derived categories of the abelian categories $R-\bmod _{I \text {-tors }}$ and $R-\bmod _{I \text {-ctra }}$,

$$
\mathrm{D}^{\star}\left(R-\bmod _{I \text {-tors }}\right) \simeq \mathrm{D}^{\star}\left(R-\bmod _{I \text {-ctra }}\right) .
$$

The triangulated equivalence (2) is a noncocommutative coalgebra version of the triangulated equivalence (4).

When $R$ is a finitely generated algebra over an algebraically closed field $k$ and $I$ is a maximal ideal in $R$, the equivalence (4) becomes a particular case of the equivalence (2). (See the discussion in [29, Section 0.10] and generally in the introduction to [29], where the conceptual importance of coalgebra-related considerations in the MGM duality theory is also emphasized.)

1.9. The triangulated equivalence (2) depends on an additional piece of data called a dedualizing complex of $\mathcal{C}$-D -bicomodules $\mathcal{B}^{\bullet}$. The definition of a dedualizing complex of bicomodules is dual to that of a dualizing complex of bimodules for a pair of associative rings [38, 39, 21, 3, 30].

A detailed discussion of the related philosophy can be found in the introduction to [29]. To recall it very briefly here, let us mention that an associative ring $A$ is itself a dedualizing complex of $A$ - $A$-bimodules. Given a dualizing complex of $A$ - $B$-bimodules $D^{\bullet}$ for a pair of associative rings $A$ and $B$, one constructs a triangulated equivalence between the coderived and the contraderived category of modules

$$
\mathrm{D}^{\mathrm{co}}(A-\bmod ) \simeq \mathrm{D}^{\mathrm{ctr}}(B-\bmod ),
$$


which can be called the covariant Serre-Grothendieck duality [30].

Conversely, a coalgebra $\mathcal{C}$ over a field $k$ is itself a dualizing complex of $\mathcal{C}-\mathcal{C}$-bicomodules; hence the triangulated equivalence (1). The datum of a dedualizing complex of $\mathcal{C}$-D-bicomodules allows to construct a triangulated equivalence (2).

1.10. Furthermore, a (semiassociative and semiunital) semialgebra $\mathcal{S}$ over a coalgebra $\mathcal{C}$ over a field $k$ is an algebra object in the (noncommutative, but associative and unital) tensor category of bicomodules over $\mathcal{C}$ with respect to the operation of cotensor product $\square_{\mathcal{e}}$. Just as for a coalgebra $\mathcal{C}$, there are four module categories naturally assigned to a semialgebra $\mathcal{S}$ : the left and right $\mathcal{S}$-semimodules, and the left and right $\mathcal{S}$-semicontramodules. The category of left $\mathcal{S}$-semimodules $\mathcal{S}$-simod is abelian and the forgetful functor $\mathcal{S}$-simod $\longrightarrow \mathcal{C}$-comod is exact if and only if $\mathcal{S}$ is an injective right $\mathcal{C}$-comodule. The category of left $\mathcal{S}$-semicontramodules $\mathcal{S}$-sicntr is abelian and the forgetful functor $\mathcal{S}$-sicntr $\longrightarrow \mathcal{C}$-contra is exact if and only if $\mathcal{S}$ is an injective left $\mathcal{C}$-comodule.

For any semialgebra $\mathcal{S}$ over a coalgebra $\mathcal{C}$ such that $\mathcal{S}$ is an injective left $\mathcal{C}$-comodule and an injective right $\mathcal{C}$-comodule, there is a natural equivalence between the semiderived categories of left $\mathcal{S}$-semimodules and left $\mathcal{S}$-semicontramodules [24, Sections 0.3.7 and 6.3]:

$$
\mathrm{D}^{\text {si }}(\boldsymbol{\mathcal { S }} \text {-simod }) \simeq \mathrm{D}^{\text {si }}(\boldsymbol{\mathcal { S }} \text {-sicntr })
$$

The words "semiderived category" actually mean two dual constructions rather than one: the semiderived category of semimodules is what could be more precisely called their semicoderived category, while the semiderived category of semicontramodules could be called the semicontraderived category. These are certain mixtures of the constructions of co- or contraderived categories (taken "along $\mathcal{C}$ ") and the conventional derived category (taken "in the direction of $\mathcal{S}$ relative to $\mathcal{C}$ ").

1.11. Now let $\mathcal{S}$ be a semialgebra over a coalgebra $\mathcal{C}$ and $\mathcal{T}$ be a semialgebra over a coalgebra $\mathcal{D}$, both over the same field $k$. Let $\mathcal{B}^{\bullet}$ be a dedualizing complex of $\mathcal{C}$-D-bicomodules. In this paper we show that, given a certain further piece of data called a dedualizing complex of $\mathcal{S}$ - $\mathcal{T}$-bisemimodules $\mathcal{B}^{\bullet}$, one can construct a triangulated equivalence between the conventional derived category of left $\mathcal{S}$-semimodules and the conventional derived category of left $\mathcal{T}$-semicontramodules,

$$
\mathrm{D}(\mathcal{S} \text {-simod }) \simeq \mathrm{D}(\mathcal{T} \text {-sicntr })
$$

Moreover, there are triangulated equivalences

$$
\mathrm{D}^{\star}(\mathcal{S} \text {-simod }) \simeq \mathrm{D}^{\star}(\mathcal{T} \text {-sicntr })
$$

for all the conventional or absolute, bounded or unbounded derived category symbols $\star=\mathrm{b},+,-, \varnothing$, abs + , abs-, or abs. These results can be called the $M G M$ duality/equivalence for semialgebras.

The definition of a dedualizing complex of bisemimodules is dual to that of a dualizing complex of bicomodules for a pair of corings over associative rings [27, Section B.4]. 
1.12. The situation simplifies when the coalgebra $\mathcal{C}$ has finite homological dimension (i. e., the abelian category $\mathcal{C}$-comod has finite homological dimension or, which is equivalent, the abelian category $\mathcal{C}$-contra has finite homological dimension).

In this case, there is no difference between the semiderived category $\mathrm{D}^{\text {si }}(\mathcal{S}$-simod $)$ and the conventional derived category $\mathrm{D}(\mathcal{S}$-simod $)$, and also no difference between the semiderived category $\mathrm{D}^{\text {si }}(\mathcal{S}$-sicntr) and the conventional derived category $\mathrm{D}(\mathcal{S}$-sicntr $)$,

$$
\mathrm{D}^{\text {si }}(\boldsymbol{\mathcal { S }} \text {-simod })=\mathrm{D}(\boldsymbol{\mathcal { S }} \text {-simod }) \quad \text { and } \quad \mathrm{D}^{\text {si }}(\boldsymbol{\mathcal { S }} \text {-sicntr })=\mathrm{D}(\boldsymbol{\mathcal { S }} \text {-sicntr }) \text {. }
$$

The semialgebra $\boldsymbol{S}$ itself can be used as a dedualizing complex of $\mathcal{S}$-S $\mathcal{S}$-bisemimodules in this case, so (6) becomes an instance of (7) for $\mathcal{C}=\mathcal{D}$ and $\mathcal{S}=\mathcal{T}$.

In particular, one finds oneself in this situation in the theory of smooth duality for a $p$-adic Lie group with coefficients in a field of characteristic $p$ [33].

1.13. Finally, let us say a few words about the finiteness conditions on coalgebras, comodules, and contramodules. One of the peculiarities of coalgebras is the difference between the classes of Artinian and co-Noetherian coalgebras or comodules. Any Artinian comodule is co-Noetherian, but the converse is not generally true. For a counterexample, one can consider the cosemisimple coalgebra $\mathcal{C}$ that is the direct sum of an infinite number of copies of the coalgebra $k$ over $k$. Then $\mathcal{C}$ is a co-Noetherian C-comodule (i. e., all its quotient comodules are finitely cogenerated), but it is not an Artinian object of the abelian category $\mathcal{C}$-comod.

The finiteness conditions on coalgebras were, of course, traditionally discussed in the language of comodules $[37,6]$. Some of the dual-analogous contramodule conditions lead to equivalent conditions on the coalgebra. In particular, any coArtinian contramodule is Noetherian, but the converse is not necessarily true. A coalgebra is called right Artinian if any finitely cogenerated right comodule over it is Artinian; this is equivalent to any finitely generated left contramodule over it being co-Artinian. A coalgebra is right cocoherent if any finitely cogenerated quotient comodule of a finitely copresented right comodule over it is finitely copresented; this is equivalent to any finitely generated subcontramodule of a finitely presented left contramodule being finitely presented.

1.14. The finiteness conditions on coalgebras, comodules, and contramodules are discussed in Section 2 of the present paper. The definition of a dedualizing complex for a pair of coalgebras is presented and the triangulated equivalence (2) is constructed in Section 3. The definition of a dedualizing complex for a pair of semialgebras is spelled out and the triangulated equivalence (8) is constructed in Section 4.

We refer to the overview paper [28] and the references therein for detailed discusions of various kinds of contramodules, including first of all contramodules over coassociative coalgebras over a field. Semialgebras, semimodules, and semicontramodules are discussed in [28, Sections 2.6 and 3.5]. The structure theory of contramodules over a coalgebra over a field was studied in [24, Appendix A]. The definitions of exotic derived categories used in this paper are introduced in [27, Appendix A]; they are also briefly recalled in [29, Appendix A]. Further discussions can be found in the introductions to [29] and [30], and in the references therein. 
Acknowledgement. The material in Sections 2-3 of this paper was originally developed as a part of the paper [29], but was later excluded from [29] (to make [29] a work in commutative algebra) and moved to this separate paper. The author was supported in part by a fellowship from the Lady Davis Foundation at the Technion while working on [29] (including much of the material now forming the present paper). The author's research was supported by the Israel Science Foundation grant \# 446/15 and the Grant Agency of the Czech Republic under the grant P201/12/G028 while preparing the paper in its present form.

\section{Coalgebras with Finiteness Conditions}

This section contains a discussion of Artinian, co-Noetherian, and cocoherent coalgebras. Many of the results below are certainly not new; we present them here for the sake of completeness of the exposition.

We refer to the book [35] and the survey paper [28] for the definitions of coassociative coalgebras over fields, comodules and contramodules over them, and the related basic concepts. A discussion of cosemisimple and conilpotent coalgebras can be found in [35, Sections 9.0-1] and (with a view towards contramodules and the terminology similar to the one in this paper) in [24, Appendix A].

Let $\mathcal{C}$ be a coassociative coalgebra (with counit) over a field $k$. For any $k$-vector space $V$ the left $\mathcal{C}$-comodule $\mathcal{C} \otimes_{k} V$ is called the cofree left $\mathcal{C}$-comodule cogenerated by $V$. For any left $\mathcal{C}$-comodule $\mathcal{L}$, there is a natural isomorphism

$$
\operatorname{Hom}_{\mathcal{C}}\left(\mathcal{L}, \mathcal{C} \otimes_{k} V\right) \simeq \operatorname{Hom}_{k}(\mathcal{L}, V)
$$

where for any two left $\mathcal{C}$-comodules $\mathcal{L}$ and $\mathcal{M}$ we denote by $\operatorname{Hom}_{\mathcal{C}}(\mathcal{L}, \mathcal{M})$ the $k$-vector space of all morphisms $\mathcal{L} \longrightarrow \mathcal{M}$ in the abelian category of left $\mathcal{C}$-comodules $\mathcal{C}$-comod. Hence cofree $\mathcal{C}$-comodules are injective objects in $\mathcal{C}$-comod. Cofree $\mathcal{C}$-comodules are sufficiently many, so any injective $\mathcal{C}$-comodule is a direct summand of a cofree one. In particular, the left $\mathcal{C}$-comodule $\mathcal{C}$ is called the cofree $\mathcal{C}$-comodule with one cogenerator, and finite direct sums of copies of $\mathcal{C}$ are the finitely cogenerated cofree $\mathcal{C}$-comodules.

A coassociative coalgebra is called cosimple if it has no nonzero proper subcoalgebras. The cosimple $k$-coalgebras are precisely the dual coalgebras to simple finitedimensional $k$-algebras. A coassociative coalgebra $\mathcal{E}$ is called cosemisimple if it is a direct sum of cosimple coalgebras, or equivalently, if the category of left $\mathcal{E}$-comodules is semisimple, or if the category of right $\mathcal{E}$-comodules is semisimple.

A coassociative coalgebra without counit $\mathrm{C}^{\prime}$ is called conilpotent if for any element $c^{\prime} \in \mathcal{C}^{\prime}$ there exists an integer $n \geqslant 1$ such that $c^{\prime}$ is annihilated by the iterated coaction map $\mathrm{C}^{\prime} \longrightarrow \mathrm{C}^{\prime \otimes n+1}$. Any coassociative coalgebra $\mathcal{C}$ has a unique maximal cosemisimple subcoalgebra $\mathcal{C}^{\text {ss }} \subset \mathcal{C}$, which can be also defined as the (direct) sum of all cosimple subcoalgebras in $\mathcal{C}$, or as the minimal subcoalgebra $\mathcal{E} \subset \mathcal{C}$ for which the quotient coalgebra without counit $\mathcal{C} / \mathcal{E}$ is conilpotent [35, Sections 9.0-1].

For any subcoalgebra $\mathcal{E} \subset \mathcal{C}$ and any left $\mathcal{C}$-comodule $\mathcal{M}$, we denote by $\mathcal{E} \mathcal{M}$ the maximal $\mathcal{C}$-subcomodule in $\mathcal{M}$ whose $\mathcal{C}$-comodule structure comes from an $\mathcal{E}$-comodule 
structure. In other words, $\varepsilon \mathcal{M} \subset \mathcal{M}$ is the full preimage of the subspace $\mathcal{E} \otimes_{k} \mathcal{M} \subset$ $\mathcal{C} \otimes_{k} \mathcal{M}$ under the left coaction map $\mathcal{M} \longrightarrow \mathcal{C} \otimes_{k} \mathcal{M}$. The following assertion is a dual version of Nakayama's lemma for comodules.

Lemma 2.1. Let $\mathcal{E} \subset \mathcal{C}$ be a subcoalgebra such that the quotient coalgebra without counit $\mathcal{C} / \mathcal{E}$ is conilpotent (i. e., $\mathcal{E}$ contains the subcoalgebra $\mathcal{e}^{\mathrm{ss}} \subset \mathcal{C}$ ). Then the subcomodule $\mathcal{E} \mathcal{M}$ is nonzero for any nonzero left $\mathcal{C}$-comodule $\mathcal{M}$.

Proof. It follows from the conilpotency condition that for every element $x \in \mathcal{M}$ there exists an integer $n \geqslant 1$ such that $x$ is annihilated by the iterated coaction map $\mathcal{M} \longrightarrow(\mathcal{C} / \mathcal{E})^{\otimes n} \otimes_{k} \mathcal{M}$. Hence the coaction map $\mathcal{M} \longrightarrow \mathcal{C} / \mathcal{E} \otimes_{k} \mathcal{M}$ cannot be injective for a nonzero left $\mathcal{C}$-comodule $\mathcal{M}$.

A left $\mathcal{C}$-comodule is said to be finitely cogenerated [36, Example 1.2] if it can be embedded as a subcomodule into a finitely cogenerated cofree left $\mathcal{C}$-comodule. Obviously, any subcomodule of a finitely cogenerated cofree $\mathcal{C}$-comodule is finitely cogenerated. One easily checks that the class of finitely cogenerated left $\mathcal{C}$-comodules is closed under extensions in $\mathrm{C}$-comod.

Lemma 2.2. (a) For any finitely cogenerated left $\mathcal{C}$-comodule $\mathcal{L}$ and any subcoalgebra $\mathcal{E} \subset \mathcal{C}$, the left $\mathcal{E}$-comodule $\varepsilon \mathcal{L}$ is finitely cogenerated.

(b) The cofree left $\mathcal{C}$-comodule $\mathcal{C} \otimes_{k} V$ with an infinite-dimensional vector space of cogenerators $V$ over a nonzero coalgebra $\mathcal{C}$ is not finitely cogenerated.

(c) For any subcoalgebra $\mathcal{E} \subset \mathcal{C}$, a left $\mathcal{E}$-comodule $\mathcal{L}$ is finitely cogenerated if and only if it is finitely cogenerated as a left $\mathcal{C}$-comodule.

(d) Let $\mathcal{E} \subset \mathcal{C}$ be a subcoalgebra such that the quotient coalgebra without counit $\mathcal{C} / \mathcal{E}$ is conilpotent. Then a left $\mathcal{C}$-comodule $\mathcal{L}$ is finitely cogenerated if and only if the left $\mathcal{E}$-comodule $\mathcal{L}$ is finitely cogenerated.

(e) A left $\mathcal{C}$-comodule $\mathcal{L}$ is finitely cogenerated if and only if the left $\mathcal{E}$-comodule $\mathcal{L}$ for every cosimple subcoalgebra $\mathcal{E} \subset \mathcal{C}$ is a finite direct sum of copies of the irreducible left $\mathcal{E}$-comodule with the multiplicity of the irreducible left $\mathcal{E}$-comodule in $\mathcal{E} \mathcal{L}$ divided by its multiplicity in the left $\mathcal{E}$-comodule $\mathcal{E}$ bounded by a single constant uniformly over all the cosimple subcoalgebras $\mathcal{E} \subset \mathcal{C}$.

Proof. Part (a): obviously, for any injective morphism of left $\mathcal{C}$-comodules $\mathcal{L} \longrightarrow \mathcal{M}$, the induced morphism $\varepsilon_{\varepsilon} \mathcal{L} \longrightarrow \varepsilon_{\varepsilon} \mathcal{M}$ is also injective, so it remains to notice the natural isomorphism of $\mathcal{E}$-comodules ${ }_{\mathcal{E}}\left(\mathcal{C} \otimes_{k} V\right) \simeq \mathcal{E} \otimes_{k} V$ for any $k$-vector space $V$. Now it suffices to pick any nonzero finite-dimensional subcoalgebra $\mathcal{E} \subset \mathcal{C}$ in order to deduce part (b) from the latter isomorphism and part (a). Part (c) follows from the same isomorphism.

Part (d): a morphism of $\mathcal{C}$-comodules $\mathcal{L} \longrightarrow \mathcal{C} \otimes_{k} V$ is uniquely determined by its composition with the map $\mathcal{C} \otimes_{k} V \longrightarrow V$ induced by the counit map $\mathcal{C} \longrightarrow k$ of the coalgebra $\mathcal{C}$; and this composition can be an arbitrary $k$-linear map $\mathcal{L} \longrightarrow V$. Suppose that we are given an injective morphism of $\mathcal{E}$-comodules $\mathcal{E} \mathcal{L} \longrightarrow \mathcal{E} \otimes_{k} V$, where $V$ is a finite-dimensional vector space. Consider the composition $\varepsilon \mathcal{L} \longrightarrow \mathcal{E} \otimes_{k} V \longrightarrow V$ and extend it arbitrarily to a $k$-linear map $\mathcal{L} \longrightarrow V$. The corresponding $\mathcal{C}$-comodule 
morphism $\mathcal{L} \longrightarrow \mathcal{C} \otimes_{k} V$ forms a commutative diagram with the injective morphism $\varepsilon \mathcal{L} \longrightarrow \mathcal{E} \otimes_{k} V$ and the embeddings $\varepsilon \mathcal{L} \longrightarrow \mathcal{L}$ and $\mathcal{E} \otimes_{k} V \longrightarrow \mathcal{C} \otimes_{k} V$. Denote by $\mathcal{K}$ the kernel of the morphism $\mathcal{L} \longrightarrow \mathcal{C} \otimes_{k} V$; then the submodule $\mathcal{K} \subset \mathcal{L}$ does not intersect the submodule ${ }_{\varepsilon} \mathcal{L} \subset \mathcal{L}$, so one has ${ }_{\varepsilon} \mathcal{K}=0$. By Lemma 2.1, it follows that $\mathcal{K}=0$. To prove part (e), one applies part $(\mathrm{d})$ to the subcoalgebra $\mathrm{e}^{\mathrm{ss}} \subset \mathcal{C}$ and then decomposes $\mathcal{C}^{\text {ss }}$ into a direct sum of its cosimple subcoalgebras $\mathcal{E}$.

A $\mathrm{C}$-comodule is called co-Noetherian if all its quotient $\mathrm{C}$-comodules are finitely cogenerated [37]. The class of co-Noetherian left $\mathcal{C}$-comodules is closed under subobjects, quotient objects, and extensions in the abelian category $\mathcal{C}$-comod [37, Proposition 4], so co-Noetherian left $\mathcal{C}$-comodules form an abelian category. Given a subcoalgebra $\mathcal{E} \subset \mathcal{C}$, an $\mathcal{E}$-comodule is co-Noetherian if and only if it is co-Noetherian as a $\mathcal{C}$-comodule.

A $\mathcal{C}$-comodule is called Artinian if every descending chain of its subcomodules terminates. As the class of Artinian objects in any abelian category, the class of Artinian left $\mathcal{C}$-comodules is closed under subobjects, quotient objects, and extensions in the abelian category $\mathcal{C}$-comod, so Artinian left $\mathcal{C}$-comodules form an abelian category. Given a subcoalgebra $\mathcal{E} \subset \mathcal{E}$, an $\mathcal{E}$-comodule is Artinian if and only if it is Artinian as a $\mathcal{C}$-comodule.

Lemma 2.3. (a) Any Artinian $\mathrm{C}$-comodule is co-Noetherian.

(b) If the subcoalgebra $\mathrm{C}^{\mathrm{ss}} \subset \mathrm{C}$ is finite-dimensional, then any co-Noetherian e-comodule is Artinian.

Proof. This is a subset of results of [6, Proposition 2.5]. Part (a): it suffices to show that any Artinian left $\mathcal{C}$-comodule $\mathcal{L}$ is finitely cogenerated. Pick a nonzero linear function $\phi_{1}: \mathcal{L} \longrightarrow k$ and consider the related morphism of left $\mathcal{C}$-comodules $f_{1}: \mathcal{L} \longrightarrow \mathcal{C}$. Let $\mathcal{L}_{1} \subset \mathcal{L}$ denote the kernel of the morphism $f_{1}$. Pick a nonzero linear function $\mathcal{L}_{1} \longrightarrow k$ and extend it to a linear function $\phi_{2}: \mathcal{L} \longrightarrow k$. Consider the related morphism of left $\mathcal{C}$-comodules $f_{2}: \mathcal{L} \longrightarrow \mathcal{C}$; let $\mathcal{L}_{2} \subset \mathcal{L}_{1}$ denote the intersection of the kernels of the morphisms $f_{1}$ and $f_{2}$, etc. According to the descending chain condition, this process must terminate, which can only happen if the intersection of the kernels of the morphisms $f_{1}, \ldots, f_{n}$ is zero for some integer $n$. We have constructed an injective morphism of left $\mathcal{C}$-comodules $\mathcal{L} \longrightarrow \mathcal{C}^{\oplus n}$.

Part (b): it suffices to show that any descending chain of subcomodules $\mathcal{L} \supset \mathcal{L}_{1} \supset$ $\mathcal{L}_{2} \supset \cdots$ with zero intersection $\bigcap_{n} \mathcal{L}_{n}=0$ terminates in a finitely cogenerated left $\mathcal{C}$-comodule $\mathcal{L}$. Indeed, by Lemma 2.2(a) together with the assumption of part (b) the subcomodule ess $\mathcal{L} \subset \mathcal{L}$ is finite-dimensional. Hence the chain of intersections ${ }_{\text {ess }} \mathcal{L} \cap \mathcal{L}_{i}$ stabilizes, and consequently, eventually vanishes, i. e., there exists $n$ for which ess $\mathcal{L} \cap \mathcal{L}_{n}=0$. Then it follows from Lemma 2.1 that $\mathcal{L}_{n}=0$.

A left contramodule $\mathfrak{P}$ over a coassociative coalgebra $\mathcal{D}$ over a field $k$ is a $k$-vector space endowed with a left $\mathcal{D}$-contraaction map $\operatorname{Hom}_{k}(\mathcal{D}, \mathfrak{P}) \longrightarrow \mathfrak{P}$ satisfying the appropriate contraassociativity and contraunitality equations. Specifically, the two $\operatorname{maps}_{\operatorname{Hom}_{k}}\left(\mathcal{D}, \operatorname{Hom}_{k}(\mathcal{D}, \mathfrak{P}) \simeq \operatorname{Hom}_{k}\left(\mathcal{D} \otimes_{k} \mathcal{D}, \mathfrak{P}\right) \rightrightarrows \operatorname{Hom}_{k}(\mathcal{D}, \mathfrak{P})\right.$ induced by the comultiplication map $\mathcal{D} \longrightarrow \mathcal{D} \otimes_{k} \mathcal{D}$ and the contraaction map should have equal 
compositions with the contraaction map $\operatorname{Hom}_{k}(\mathcal{D}, \mathfrak{P}) \longrightarrow \mathfrak{P}$,

$$
\operatorname{Hom}_{k}\left(\mathcal{D}, \operatorname{Hom}_{k}(\mathcal{D}, \mathfrak{P})\right) \simeq \operatorname{Hom}_{k}\left(\mathcal{D} \otimes_{k} \mathcal{D}, \mathfrak{P}\right) \rightrightarrows \operatorname{Hom}_{k}(\mathcal{D}, \mathfrak{P}) \longrightarrow \mathfrak{P}
$$

while the composition of the map $\mathfrak{P} \longrightarrow \operatorname{Hom}_{k}(\mathcal{D}, \mathfrak{P})$ induced by the counit map $\mathcal{D} \longrightarrow k$ with the contraaction map should be equal to the identity map on the contramodule $\mathfrak{P}$,

$$
\mathfrak{P} \longrightarrow \operatorname{Hom}_{k}(\mathcal{D}, \mathfrak{P}) \longrightarrow \mathfrak{P}
$$

The natural isomorphism $\operatorname{Hom}_{k}\left(U, \operatorname{Hom}_{k}(V, W)\right) \simeq \operatorname{Hom}_{k}\left(V \otimes_{k} U, W\right)$ connecting the tensor product and Hom functors on the category of $k$-vector spaces is presumed in the first equation.

Left $\mathcal{D}$-contramodules form an abelian category $\mathcal{D}$-contra with an exact forgetful functor to the category of $k$-vector spaces $\mathcal{D}$-contra $\longrightarrow k$-vect, preserving infinite products but not infinite direct sums (see [28, Sections 1.1-1.2] and the references therein). For any right $\mathcal{D}$-comodule $\mathcal{N}$ and $k$-vector space $V$, the vector space $\operatorname{Hom}_{k}(\mathcal{N}, V)$ has a natural left $\mathcal{D}$-contramodule structure. In particular, the left $\mathcal{D}$-contramodule $\operatorname{Hom}_{k}(\mathcal{D}, V)$ is called the free left $\mathcal{D}$-contramodule generated by $V$. For any left $\mathcal{D}$-contramodule $\mathfrak{Q}$, there is a natural isomorphism

$$
\operatorname{Hom}^{\mathcal{D}}\left(\operatorname{Hom}_{k}(\mathcal{D}, V), \mathfrak{Q}\right) \simeq \operatorname{Hom}_{k}(V, \mathfrak{Q}),
$$

where for any two left $\mathcal{D}$-contramodules $\mathfrak{P}$ and $\mathfrak{Q}$ we denote by $\operatorname{Hom}^{\mathcal{D}}(\mathfrak{P}, \mathfrak{Q})$ the $k$-vector space of all morphisms $\mathfrak{P} \longrightarrow \mathfrak{Q}$ in the abelian category $\mathcal{D}$-contra. Hence free $\mathcal{D}$-contramodules are projective objects in $\mathcal{D}$-contra. There are enough of them, so any projective left $\mathcal{D}$-contramodule is a direct summand of a free one. The left $\mathcal{D}$-contramodule $\operatorname{Hom}_{k}(\mathcal{D}, k)$ is called the free $\mathcal{D}$-contramodule with one generator, and the $\mathcal{D}$-contramodules $\operatorname{Hom}_{k}(\mathcal{D}, V)$ with finite-dimensional $k$-vector spaces $V$ are the finitely generated free $\mathcal{D}$-contramodules.

For any subcoalgebra $\mathcal{E} \subset \mathcal{D}$ and any left $\mathcal{D}$-contramodule $\mathfrak{P}$, we denote by ${ }^{\varepsilon_{\mathfrak{P}}}$ the maximal quotient $\mathcal{D}$-contramodule of $\mathfrak{P}$ whose $\mathcal{D}$-contramodule structure comes from an $\mathcal{E}$-contramodule structure. In other words, ${ }^{\varepsilon} \mathfrak{P}$ is the cokernel of the composition $\operatorname{Hom}_{k}(\mathcal{D} / \mathcal{E}, \mathfrak{P}) \longrightarrow \mathfrak{P}$ of the embedding $\operatorname{Hom}_{k}(\mathcal{D} / \mathcal{E}, \mathfrak{P}) \longrightarrow \operatorname{Hom}_{k}(\mathcal{D}, \mathfrak{P})$ with the contraaction map $\operatorname{Hom}_{k}(\mathcal{D}, \mathfrak{P}) \longrightarrow \mathfrak{P}$. The following assertion is called the Nakayama lemma for contramodules over coalgebras over fields.

Lemma 2.4. Let $\mathcal{E} \subset \mathcal{D}$ be a subcoalgebra such that the quotient coalgebra without counit $\mathcal{D} / \mathcal{E}$ is conilpotent. Then the quotient contramodule ${ }^{\varepsilon} \mathfrak{P}$ is nonzero for any nonzero left $\mathcal{D}$-contramodule $\mathfrak{P}$.

Proof. This is [24, Lemma A.2.1]; see also [26, Lemma 1.3.1] and [28, Lemma 2.1].

A left $\mathcal{D}$-contramodule is said to be finitely generated if it is a quotient contramodule of a finitely generated free left $\mathcal{D}$-contramodule. The class of finitely generated left $\mathcal{D}$-contramodules is closed under extensions and the passages to quotient objects.

Lemma 2.5. (a) For any finitely generated left $\mathcal{D}$-contramodule $\mathfrak{Q}$ and any subcoalgebra $\mathcal{E} \subset \mathcal{D}$, the left $\mathcal{E}$-contramodule ${ }^{\mathcal{E}} \mathfrak{Q}$ is finitely generated. 
(b) The free $\mathcal{D}$-contramodule $\operatorname{Hom}_{k}(\mathcal{D}, V)$ with an infinite-dimensional vector space of generators $V$ over a nonzero coalgebra $\mathcal{D}$ is not finitely generated.

(c) For any subcoalgebra $\mathcal{E} \subset \mathcal{D}$, a left $\mathcal{E}$-contramodule is finitely generated if and only if it is finitely generated as a left $\mathcal{D}$-contramodule.

(d) Let $\mathcal{E} \subset \mathcal{D}$ be a subcoalgebra such that the quotient coalgebra without counit $\mathcal{D} / \mathcal{E}$ is conilpotent. Then a left $\mathcal{D}$-contramodule $\mathfrak{Q}$ is finitely generated if and only if the left $\mathcal{E}$-contramodule ${ }^{\mathcal{E}} \mathfrak{Q}$ is finitely generated.

(e) A left $\mathcal{D}$-contramodule $\mathfrak{Q}$ is finitely generated if and only if the left $\mathcal{E}$-contramodule ${ }^{\mathcal{E}} \mathfrak{Q}$ for every cosimple subcoalgebra $\mathcal{E} \subset \mathcal{D}$ is a finite direct sum of copies of the irreductive left $\mathcal{E}$-contramodule with the multiplicity of the irreductible left $\mathcal{E}$-contramodule in ${ }^{\varepsilon} \mathfrak{Q}$ divided by its multiplicity in the left $\mathcal{E}$-contramodule $\mathcal{E}^{*}=\operatorname{Hom}_{k}(\mathcal{E}, k)$ bounded by a single constant uniforly over all the cosimple subcoalgebras $\mathcal{E} \subset \mathcal{D}$.

Proof. The proof is dual-analogous to that of Lemma 2.2. To prove parts (a-c), one notices the natural isomorphism ${ }^{\varepsilon} \operatorname{Hom}_{k}(\mathcal{D}, V) \simeq \operatorname{Hom}_{k}(\mathcal{E}, V)$ for any subcoalgebra $\mathcal{E} \subset \mathcal{D}$ and $k$-vector space $V$. Part (d): given a surjective morphism of $\mathcal{E}$-contramodules $\operatorname{Hom}_{k}(\mathcal{E}, V) \longrightarrow{ }^{\mathcal{E}} \mathfrak{Q}$ with a finite-dimensional vector space $V$, one considers the composition $V \longrightarrow \operatorname{Hom}_{k}(\mathcal{E}, V) \longrightarrow{ }^{\varepsilon} \mathfrak{Q}$ and lifts it to a $k$-linear map $V \longrightarrow \mathfrak{Q}$. The corresponding morphism of $\mathcal{D}$-contramodules $\operatorname{Hom}_{k}(\mathcal{D}, V) \longrightarrow \mathfrak{Q}$ is surjective by Lemma 2.4, since one has ${ }^{\mathcal{E}_{\mathfrak{K}}}=0$ for its cokernel $\mathfrak{K}$. To prove part (e), one applies part $(\mathrm{d})$ to the subcoalgebra $\mathcal{D}^{\text {ss }} \subset \mathcal{D}$ and applies [24, Lemma A.2.2] in order to decompose the $\mathcal{D}^{\text {ss }}$-contramodule ${ }^{\mathcal{D}^{\text {ss }}} \mathfrak{Q}$ into a product of contramodules over the cosimple subcoalgebras $\mathcal{E} \subset \mathcal{D}^{\text {ss }}$.

A left $\mathcal{D}$-contramodule is called Noetherian if all its subcontramodules are finitely generated. The class of Noetherian left $\mathcal{D}$-contramodules is closed under subobjects, quotient objects, and extensions in the abelian category $\mathcal{D}$-contra, so Noetherian left $\mathcal{D}$-contramodules form an abelian category. Given a subcoalgebra $\mathcal{E} \subset \mathcal{D}$, an $\mathcal{E}$-contramodule is Noetherian if and only if it is Noetherian as a $\mathcal{D}$-contramodule.

A $\mathcal{D}$-contramodule is called co-Artinian if every ascending chain of its subcontramodules terminates. As the similar class of objects in any abelian category, the class of co-Artinian left $\mathcal{D}$-contramodules is closed under subobjects, quotient objects, and extensions in the abelian category $\mathcal{D}$-contra, so co-Artinian left $\mathcal{D}$-contramodules form an abelian category. Given a subcoalgebra $\mathcal{E} \subset \mathcal{D}$, an $\mathcal{E}$-contramodule is coArtinian if and only if it is co-Artinian as a $\mathcal{D}$-contramodule.

Lemma 2.6. (a) Any co-Artinian D-contramodule is Noetherian.

(b) If the subcoalgebra $\mathcal{D}^{\mathrm{ss}} \subset \mathcal{D}$ is finite-dimensional, then any Noetherian $\mathcal{D}$-contramodule is co-Artinian.

Proof. Part (a): it suffices to show that any co-Artinian left $\mathcal{D}$-contramodule $\mathfrak{Q}$ is finitely generated. Pick an element $q_{1} \in \mathfrak{Q}$ and consider the related morphism of left $\mathcal{D}$-contramodules $f_{1}: \mathcal{D}^{*}=\operatorname{Hom}_{k}(\mathcal{D}, k) \longrightarrow \mathfrak{Q}$. Pick an element $q_{2} \in \mathfrak{Q}$ outside of the image of $f_{1}$, consider the related morphism $f_{2}: \mathcal{D}^{*} \longrightarrow \mathfrak{Q}$, pick an element $q_{3} \in \mathfrak{Q}$ outside of the sum of the images of $f_{1}$ and $f_{2}$, etc. According to the ascending chain condition, this process must terminate, which means that the sum of the images of 
the morphisms $f_{1}, \ldots, f_{n}$ is the whole of $\mathfrak{Q}$ for some integer $n$. We have constructed as surjective morphism of left $\mathcal{D}$-contramodules $\mathcal{D}^{* \oplus n} \longrightarrow \mathfrak{Q}$.

Part (b): it suffices to show that an ascending chain of subcontramodules $\mathfrak{Q}_{1} \subset$ $\mathfrak{Q}_{2} \subset \cdots \subset \mathfrak{Q}$ terminates in a finitely generated left $\mathcal{D}$-contramodule $\mathfrak{Q}$ provided that there is no proper subcontramodule in $\mathfrak{Q}$ containing all the subcontramodules $\mathfrak{Q}_{n}$. Indeed, by Lemma 2.5(a) together with the assumption of part (b) the maximal quotient $\mathcal{D}^{\text {ss }}$-contramodule ${ }^{D^{\text {ss }}} \mathfrak{Q}$ of the $\mathcal{D}$-contramodule $\mathfrak{Q}$ is finite-dimensional. Hence the chain of the images of the subcontramodules $\mathfrak{Q}_{n} \subset \mathfrak{Q}$ in ${ }^{D^{s s}} \mathfrak{Q}$ stabilizes, and consequently, eventually reaches the whole of ${ }^{D^{s s}} \mathfrak{Q}$, i. e., there exists $n$ for which the composition $\mathfrak{Q}_{n} \longrightarrow \mathfrak{Q} \longrightarrow{ }^{D^{s s}} \mathfrak{Q}$ is surjective. Then one has ${ }^{{ }^{s s}}\left(\mathfrak{Q} / \mathfrak{Q}_{n}\right)=0$, and it follows from Lemma 2.4 that $\mathfrak{Q}_{n}=\mathfrak{Q}$.

Example 2.7. Let $\mathcal{C}$ be an infinite-dimensional cosemisimple coalgebra. Then the left $\mathcal{C}$-comodule $\mathcal{C}$ is co-Noetherian, but not Artinian. Similarly, the left $\mathcal{C}$-contramodule $\mathcal{C}^{*}$ is Noetherian, but not co-Artinian. It follows that the classes of Artinian and co-Noetherian left comodules over a coalgebra $\mathcal{D}$ coincide if and only if the classes of co-Artinian and Noetherian left contramodules over $\mathcal{D}$ coincide and if and only if the subcoalgebra $\mathcal{D}^{\text {ss }} \subset \mathcal{D}$ is finite-dimensional.

A left $\mathcal{D}$-contramodule is said to be finitely presented if it is the cokernel of a morphism of finitely generated free left $\mathcal{D}$-contramodules. Clearly, the cokernel of a morphism from a finitely generated left $\mathcal{D}$-contramodule to a finitely presented one is finitely presented. It is easy to check that an extension of finitely presented left $\mathcal{D}$-contramodules is finitely presented.

A left $\mathcal{C}$-comodule is said to be finitely copresented if it is the kernel of a morphism of finitely cogenerated cofree $\mathcal{C}$-comodules. Clearly, the kernel of a morphism from a finitely copresented left $\mathcal{C}$-comodule to a finitely cogenerated one is finitely copresented; an extension of finitely copresented left $\mathcal{C}$-comodules is finitely copresented.

Part (a) of the next lemma can be found in [37, Theorem 6].

Lemma 2.8. (a) The cokernel of an injective morphism from a finitely copresented C-comodule to a finitely cogenerated one is finitely cogenerated.

(b) The kernel of a surjective morphism from a finitely generated $\mathcal{D}$-contramodule to a finitely presented one is finitely generated.

Proof. Part (a): let $\mathcal{L}$ be the kernel of a morphism of finitely cogenerated cofree $\mathcal{C}$-comodules $\mathcal{J} \longrightarrow \mathcal{J}$, let $\mathcal{M}$ be a finitely cogenerated $\mathcal{C}$-comodule, and let $\mathcal{L} \longrightarrow$ $\mathcal{M}$ be an injective morphism with the cokernel $\mathcal{K}$. Denote by $\mathcal{N}$ the fibered coproduct of $\mathcal{C}$-comodules $\mathcal{J}$ and $\mathcal{M}$ over the $\mathcal{C}$-comodule $\mathcal{L}$; then there are exact sequences of $\mathrm{C}$-comodules $0 \longrightarrow \mathcal{N} \longrightarrow \mathcal{N} \longrightarrow \mathcal{J}$ and $0 \longrightarrow \mathcal{J} \longrightarrow \mathcal{N} \longrightarrow \mathcal{K} \longrightarrow 0$. Now the $\mathcal{C}$-comodule $\mathcal{N}$ is finitely cogenerated as an extension of finitely cogenerated $\mathcal{C}$-comodules; and the $\mathcal{C}$-comodule $\mathcal{K}$ is a direct summand of $\mathcal{N}$, because the $\mathcal{C}$-comodule $\mathcal{J}$ is injective. The proof of part (b) is analogous.

The dual vector space $\mathcal{D}^{*}$ to a coassociative coalgebra $\mathcal{D}$ has a natural structure of topological associative algebra. There is but a slight ambiguity in its definition in 
that one has to make a decision about the order of the factors in the multiplication operation, i. e., which one of the two opposite algebras is to be denoted by $\mathcal{D}^{*}$ and which one by $\mathcal{D}^{* \mathrm{op}}$. We prefer the convention according to which right $\mathcal{D}$-comodules $\mathcal{N}$ become discrete right $\mathcal{D}^{*}$-modules; then the dual vector space $\mathcal{N}^{*}$ is a left $\mathcal{D}^{*}$-module (see [28, Sections 1.3-4] for a further discussion). Any left $\mathcal{D}$-contramodule has an underlying structure of left $\mathcal{D}^{*}$-module (see [28, Section 2.3] and [24, Section A.1.2]).

One observes that a left $\mathcal{D}$-contramodule is finitely generated if and only if its underlying left $\mathcal{D}^{*}$-module is finitely generated. It follows that a left $\mathcal{D}$-contramodule is finitely presented if and only if its underlying left $\mathcal{D}^{*}$-module is.

Proposition 2.9. (a) The restrictions of the functor $\mathcal{L} \longmapsto \mathcal{L}^{*}=\operatorname{Hom}_{k}(\mathcal{L}, k)$ and the forgetful functor $\mathcal{D}$-contra $\longrightarrow \mathcal{D}^{*}$-mod provide an anti-equivalence between the additive category of finitely copresented right $\mathcal{D}$-comodules and the additive category of finitely presented left $\mathcal{D}$-contramodules, and an isomorphism between the latter category and the additive category of finitely presented left $\mathcal{D}^{*}$-modules.

(b) For any right $\mathcal{D}$-comodule $\mathcal{N}$ and any finitely copresented right $\mathcal{D}$-comodule $\mathcal{L}$, the functor $\mathcal{N} \longmapsto \mathcal{N}^{*}=\operatorname{Hom}_{k}(\mathcal{N}, k)$ and the forgetful functor $\mathcal{D}$-contra $\longrightarrow \mathcal{D}^{*}$-mod induce isomorphisms of the Hom spaces

$$
\operatorname{Hom}_{\mathcal{D}^{\circ p}}(\mathcal{N}, \mathcal{L}) \simeq \operatorname{Hom}^{\mathcal{D}}\left(\mathcal{L}^{*}, \mathcal{N}^{*}\right) \simeq \operatorname{Hom}_{\mathcal{D}^{*}}\left(\mathcal{L}^{*}, \mathcal{N}^{*}\right)
$$

in the categories of right $\mathcal{D}$-comodules, left $\mathcal{D}$-contramodules, and left $\mathcal{D}^{*}$-modules.

Proof. Since the functor Hom preserves kernels in its second argument and transforms cokernels in its first argument into kernels, it suffices to prove part (b) for finitely generated cofree right $\mathcal{D}$-comodules $\mathcal{L}=V \otimes_{k} \mathcal{D}$, where $V$ is a finite-dimensional $k$-vector space. Then $\mathcal{L}^{*} \simeq \operatorname{Hom}_{k}\left(\mathcal{D}, V^{*}\right) \simeq \mathcal{D}^{*} \otimes_{k} V^{*}$ is a finitely generated free left $\mathcal{D}$-contramodule and a finitely generated free left $\mathcal{D}^{*}$-module. One easily computes $\operatorname{Hom}_{\mathcal{D} \text { op }}\left(\mathcal{N}, V \otimes_{k} \mathcal{D}\right) \simeq \operatorname{Hom}_{k}(\mathcal{N}, V), \operatorname{Hom}^{\mathcal{D}}\left(\operatorname{Hom}_{k}\left(\mathcal{D}, V^{*}\right), \mathcal{N}^{*}\right) \simeq \operatorname{Hom}_{k}\left(V^{*}, \mathcal{N}^{*}\right)$, and $\operatorname{Hom}_{\mathcal{D}^{*}}\left(\mathcal{D}^{*} \otimes_{k} V^{*}, \mathcal{N}^{*}\right) \simeq \operatorname{Hom}_{k}\left(V^{*}, \mathcal{N}^{*}\right)$, implying part (b). Part (a) immediately follows from the same computation of Hom spaces.

Lemma 2.10. (a) A left D-contramodule is co-Artinian if and only if it is a Noetherian left $\mathcal{D}^{*}$-module.

(b) A right $\mathcal{D}$-comodule $\mathcal{L}$ is Artinian if and only if dual vector space $\mathcal{L}^{*}$ is a Noetherian left $\mathcal{D}^{*}$-module.

(c) A right $\mathcal{D}$-comodule $\mathcal{L}$ is co-Noetherian provided that its dual vector space $\mathcal{L}^{*}$ is a Noetherian left $\mathcal{D}$-contramodule.

Proof. Part (a): one notices that a $\mathcal{D}^{*}$-module is Noetherian if and only if any ascending chain of its finitely generated submodules terminates. Similarly, a $\mathcal{D}$-contramodule is co-Artinian if and only if any ascending chain of its finitely generated subcontramodules terminates. Finally, the classes of finitely generated $\mathcal{D}^{*}$-submodules and finitely generated $\mathcal{D}$-subcontramodules in any given $\mathcal{D}$-contramodule coincide.

Part (b) is again a subset of [6, Proposition 2.5]. To any descending chain of $\mathcal{D}$-subcomodules in $\mathcal{L}$ one can assign the ascending chain of their orthogonal complements, which are $\mathcal{D}^{*}$-submodules in $\mathcal{L}^{*}$. Conversely, in view of Proposition 2.9(b), any 
finitely generated $\mathcal{D}^{*}$-submodule in $\mathcal{L}^{*}$ is the orthogonal complement to a certain $\mathcal{D}$-subcomodule in $\mathcal{L}$. Part (c): for any quotient comodule of $\mathcal{L}$, there is its dual subcontramodule in $\mathcal{L}^{*}$. It remains to notice that a right $\mathcal{D}$-comodule $\mathcal{N}$ is finitely cogenerated if and only if its dual left $\mathcal{D}$-contramodule $\mathcal{N}^{*}$ is finitely generated.

A finitely cogenerated left $\mathcal{C}$-comodule is called cocoherent if every its finitely cogenerated quotient comodule is finitely copresented. Using Lemma 2.8(a), one can show that the class of cocoherent left $\mathcal{C}$-comodules is closed under the operations of the passage to the kernels, cokernels, and extensions in the abelian category $\mathcal{C}-$ comod; so cocoherent left $\mathcal{C}$-comodules form an abelian category.

Analogously, a finitely presented left $\mathcal{D}$-contramodule is called coherent if every its finitely generated subcontramodule is finitely presented. Using Lemma 2.8(b), one shows that the class of coherent left $\mathcal{D}$-contramodules is closed under the passages to the kernels, cokernels, and extensions in the abelian category $\mathcal{D}$-comod; so coherent left $\mathcal{D}$-contramodules form an abelian category.

Lemma 2.11. (a) A left $\mathcal{D}$-contramodule is coherent if and only if its underlying left $\mathcal{D}^{*}$-module is coherent. The abelian categories of coherent left $\mathcal{D}$-contramodules and coherent left $\mathcal{D}^{*}$-modules are isomorphic.

(b) A right $\mathcal{D}$-comodule $\mathcal{L}$ is cocoherent if and only if its dual left $\mathcal{D}^{*}$-module $\mathcal{L}^{*}$ is coherent. The abelian categories of cocoherent right $\mathcal{D}$-comodules and coherent left $\mathcal{D}^{*}$-modules are anti-equivalent.

Proof. In view of Proposition 2.9(a), it suffices to check the first assertion in each of the parts (a) and (b). In part (a), one uses the bijection between finitely generated $\mathcal{D}$-subcontramodules and finitely generated $\mathcal{D}^{*}$-submodules of a given $\mathcal{D}$-contramodule, together with the fact that a $\mathcal{D}$-contramodule is finitely presented if and only if it is finitely presented as a $\mathcal{D}^{*}$-module. In part (b), one uses the bijection between finitely cogenerated quotient $\mathcal{D}$-comodules of $\mathcal{L}$ and finitely generated $\mathcal{D}^{*}$-submodules of $\mathcal{L}^{*}$, together with the fact that a $\mathcal{D}$-comodule is finitely copresented if and only if its dual $\mathcal{D}^{*}$-module is finitely presented.

A coalgebra $\mathcal{C}$ is called left co-Noetherian if any quotient comodule of a finitely cogenerated left $\mathcal{C}$-comodule is finitely cogenerated, or equivalently, if the left $\mathcal{C}$-comodule $\mathcal{C}$ is co-Noetherian [37, Theorem 3]. Over a left co-Noetherian coalgebra $\mathcal{C}$, finitely cogenerated left comodules form an abelian category. By Lemma 2.2(c), any subcoalgebra of a left co-Noetherian coalgebra is left co-Noetherian. Any cosemisimple coalgebra is left and right co-Noetherian.

A coalgebra $\mathcal{D}$ is called right Artinian if any finitely cogenerated right $\mathcal{D}$-comodule is Artinian, or equivalently, if the right $\mathcal{D}$-comodule $\mathcal{D}$ is Artinian, or if any finitely generated left $\mathcal{D}$-contramodule is co-Artinian, or if the left $\mathcal{D}$-contramodule $\mathcal{D}^{*}=$ $\operatorname{Hom}_{k}(\mathcal{D}, k)$ is co-Artinian (see Lemma 2.10(a-b) for a proof of the equivalence between the second and the fourth of these conditions). A coalgebra $\mathcal{D}$ is right Artinian if and only if its dual algebra $\mathcal{D}^{*}$ is left Noetherian. Any subcoalgebra of a right Artinian coalgebra is right Artinian. 
According to Lemma 2.3 and Example 2.7, any left Artinian coalgebra $\mathcal{C}$ is left co-Noetherian, but the converse is not generally true. More precisely, a coalgebra $\mathcal{C}$ is left Artinian if and only if it is left co-Noetherian and its maximal cosemisimple subcoalgebra $\mathcal{C}^{\mathrm{ss}} \subset \mathcal{e}$ is finite-dimensional.

Examples 2.12. The functor $\mathcal{C} \longmapsto \mathcal{C}^{*}$ is an anti-equivalence between the category of coassociative coalgebras and the category of pro-finite-dimensional topological associative algebras, so one can describe coalgebras in terms of their dual topological algebras. In particular, the topological algebra of formal Taylor power series in commuting variables $k\left[\left[z_{1}, \ldots, z_{m}\right]\right]$ corresponds to a certain cocommutative coalgebra $\mathcal{C}$. The algebra $k\left[\left[z_{1}, \ldots, z_{m}\right]\right]$ is Noetherian, so the coalgebra $\mathcal{C}$ is Artinian. Hence all the subcoalgebras of $\mathcal{C}$ are Artinian (and consequently, co-Noetherian), too. These are precisely the coalgebras dual to the topological algebras of functions on the formal completions of algebraic varieties over $k$ at their closed points defined over $k$. Given a field extension $k \subset \ell$, a coalgebra $\mathcal{C}$ over the field $k$ is Artinian or co-Noetherian whenever the coalgebra $\ell \otimes_{k} \mathrm{C}$ over the field $\ell$ is. Hence it follows that all the coalgebras dual to the topological algebras of functions on the formal completions of varieties over $k$ at their closed points are Artinian.

Moreover, there are many noncocommutative Artinian coalgebras, like, e. g., the coalgebra dual to the algebra of quantum formal power series $k\left\{\left\{z_{1}, \cdots, z_{m}\right\}\right\}$ with the relations $z_{i} z_{j}=q_{i, j} z_{j} z_{i}$ for all $i<j$, with any constants $q_{i, j} \in k^{*}$.

A coalgebra $\mathcal{D}$ is called right cocoherent if any finitely cogenerated quotient comodule of a finitely copresented right $\mathcal{D}$-comodule is finitely copresented, or equivalently, if the right $\mathcal{D}$-comodule $\mathcal{D}$ is cocoherent. Equivalently, a coalgebra $\mathcal{D}$ is right cocoherent if any finitely generated subcontramodule of a finitely presented left $\mathcal{D}$-contramodule is finitely presented, or if the left $\mathcal{D}$-contramodule $\mathcal{D}^{*}$ is coherent. Over a right cocoherent coalgebra $\mathcal{D}$, both the finitely copresented right $\mathcal{D}$-comodules and the finitely presented left $\mathcal{D}$-contramodules form abelian categories. A coalgebra $\mathcal{D}$ is right cocoherent if and only if its dual algebra $\mathcal{D}^{*}$ is left coherent (see Lemma 2.11). Any left co-Noetherian coalgebra $\mathcal{C}$ is left cocoherent, and any finitely cogenerated left $\mathcal{C}$-comodule is finitely copresented.

The contratensor product $\mathcal{N} \odot_{\mathcal{D}} \mathfrak{P}$ of a right $\mathcal{D}$-comodule $\mathcal{N}$ and a left $\mathcal{D}$-contramodule $\mathfrak{P}[28$, Section 3.1] is a $k$-vector space constructed as the cokernel of (the difference of) the pair of maps

$$
\mathcal{N} \otimes_{k} \operatorname{Hom}_{k}(\mathcal{D}, \mathfrak{P}) \rightrightarrows \mathcal{N} \otimes_{k} \mathfrak{P},
$$

one which is induced by the $\mathcal{D}$-contraaction in $\mathfrak{P}$, while the other one is the composition $\mathcal{N} \otimes_{k} \operatorname{Hom}_{k}(\mathcal{D}, \mathfrak{P}) \longrightarrow \mathcal{N} \otimes_{k} \mathcal{D} \otimes_{k} \operatorname{Hom}_{k}(\mathcal{D}, \mathfrak{P}) \longrightarrow \mathcal{N} \otimes_{k} \mathfrak{P}$ of the map induced by the right $\mathcal{D}$-coaction map $\mathcal{N} \longrightarrow \mathcal{N} \otimes_{k} \mathcal{D}$ and the map induced by the evaluation map $\mathcal{D} \otimes_{k} \operatorname{Hom}_{k}(\mathcal{D}, \mathfrak{P}) \longrightarrow \mathfrak{P}$. The functor of contratensor product of comodules and contramodules over a coalgebra $\mathcal{D}$ is right exact. 
For any right $\mathcal{D}$-comodule $\mathcal{N}$ and a $k$-vector space $V$ there is a natural isomorphism of $k$-vector spaces

$$
\mathcal{N} \odot_{\mathcal{D}} \operatorname{Hom}_{k}(\mathcal{D}, V) \simeq \mathcal{N} \otimes_{k} V
$$

while for any right $\mathcal{D}$-comodule $\mathcal{N}$, any left $\mathcal{D}$-contramodule $\mathfrak{P}$, and a $k$-vector space $V$ there is a natural isomorphism of $k$-vector spaces

$$
\operatorname{Hom}_{k}\left(\mathcal{N} \odot_{\mathcal{D}} \mathfrak{P}, V\right) \simeq \operatorname{Hom}^{\mathcal{D}}\left(\mathfrak{P}, \operatorname{Hom}_{k}(\mathcal{N}, V)\right)
$$

The cotensor product $\mathcal{N} \square_{\mathcal{e}} \mathcal{M}$ of a right $\mathcal{C}$-comodule $\mathcal{N}$ and a left $\mathcal{C}$-comodule $\mathcal{M}$ [28, Sections 2.5-6] is a $k$-vector space constructed as the kernel of the pair of maps

$$
\mathcal{N} \otimes_{k} \mathcal{M} \rightrightarrows \mathcal{N} \otimes_{k} \mathcal{C} \otimes_{k} \mathcal{M}
$$

one of which is induced by the right $\mathcal{C}$-coaction in $\mathcal{N}$ and the other one by the left $\mathcal{C}$-coaction in $\mathcal{M}$. The functor of cotensor product of comodules over a coalgebra $\mathcal{C}$ is left exact.

For any right $\mathcal{C}$-comodule $\mathcal{N}$, left $\mathcal{C}$-comodule $\mathcal{M}$, and $k$-vector space $V$ there are natural isomorphisms of $k$-vector spaces

$$
\mathcal{N} \square_{\mathcal{C}}\left(\mathcal{C} \otimes_{k} V\right) \simeq \mathcal{N} \otimes_{k} V \quad \text { and } \quad\left(V \otimes_{k} \mathcal{C}\right) \square_{\mathcal{C}} \mathcal{M} \simeq V \otimes_{k} \mathcal{M} .
$$

For any left $\mathcal{C}$-comodule $\mathcal{M}$ and any subcoalgebra $\mathcal{E} \subset \mathcal{C}$ there is a natural isomorphism of left $\mathcal{E}$-comodules

$$
{ }_{\varepsilon} \mathcal{M} \simeq \mathcal{E} \square_{\mathcal{e}} \mathcal{M}
$$

where the left $\mathcal{E}$-comodule structure on the cotensor product is induced by the left $\mathcal{E}$-comodule structure on $\mathcal{E}$.

The $k$-vector space of cohomomorphisms $\operatorname{Cohom}_{\mathcal{D}}(\mathcal{M}, \mathfrak{P})$ from a left $\mathcal{C}$-comodule $\mathcal{M}$ to a left $\mathcal{D}$-contramodule $\mathfrak{P}$ is a $k$-vector space constructed as the cokernel of the pair of maps

$$
\operatorname{Hom}_{k}\left(\mathcal{D} \otimes_{k} \mathcal{M}, \mathfrak{P}\right) \simeq \operatorname{Hom}_{k}\left(\mathcal{M}, \operatorname{Hom}_{k}(\mathcal{D}, \mathfrak{P})\right) \rightrightarrows \operatorname{Hom}_{k}(\mathcal{M}, \mathfrak{P}),
$$

one of which is induced by the left $\mathcal{D}$-coaction in $\mathcal{M}$ and the other one by the left $\mathcal{D}$-contraaction in $\mathfrak{P}$. The functor of cohomomorphisms from left comodules to left contramodules over a coalgebra $\mathcal{D}$ is right exact.

For any left $\mathcal{D}$-comodule $\mathcal{M}$, left $\mathcal{D}$-contramodule $\mathfrak{P}$, and $k$-vector space $V$ there are natural isomorphisms of $k$-vector spaces

$\operatorname{Cohom}_{\mathcal{D}}\left(\mathcal{D} \otimes_{k} V, \mathfrak{P}\right) \simeq \operatorname{Hom}_{k}(V, \mathfrak{P})$ and $\operatorname{Cohom}_{\mathcal{D}}\left(\mathcal{M}, \operatorname{Hom}_{k}(\mathcal{D}, V)\right) \simeq \operatorname{Hom}_{k}(\mathcal{M}, V)$.

For any left $\mathcal{D}$-contramodule $\mathfrak{P}$ and any subcoalgebra $\mathcal{E} \subset \mathcal{D}$ there is a natural isomorphism of left $\mathcal{E}$-contramodules

$$
{ }^{\varepsilon} \mathfrak{P} \simeq \operatorname{Cohom}_{\mathcal{D}}(\mathcal{E}, \mathfrak{P})
$$

where the left $\mathcal{E}$-contramodule structure on the Cohom space is induced by the right $\mathcal{E}$-comodule structure on $\mathcal{E}$. 
Lemma 2.13. For any right $\mathcal{D}$-comodule $\mathcal{N}$ and any left $\mathcal{D}$-contramodule $\mathfrak{P}$ there is a natural surjective map of $k$-vector spaces from the tensor product over the algebra $\mathcal{D}^{*}$ to the contratensor product over the coalgebra $\mathcal{D}$

$$
\mathcal{N} \otimes_{\mathcal{D}^{*}} \mathfrak{P} \longrightarrow \mathcal{N} \odot_{\mathcal{D}} \mathfrak{P}
$$

This map is an isomorphism, at least, whenever either

(a) the left $\mathcal{D}$-contramodule $\mathfrak{P}$ is finitely presented, or

(b) the coalgebra $\mathcal{D}$ is left co-Noetherian.

Proof. To construct the surjective $k$-linear map in question, one notices that the tensor product $\mathcal{N} \otimes_{\mathcal{D}^{*}} \mathfrak{P}$ is the cokernel of a natural map $\mathcal{N} \otimes_{k} \mathcal{D}^{*} \otimes_{k} \mathfrak{P} \longrightarrow \mathcal{N} \otimes_{k} \mathfrak{P}$, while the contratensor product $\mathcal{N} \square_{\mathcal{D}} \mathfrak{P}$ is the cokernel of a map $\mathcal{N} \otimes_{k} \operatorname{Hom}_{k}(\mathcal{D}, \mathfrak{P}) \longrightarrow$ $\mathcal{N} \otimes_{k} \mathfrak{P}$. These two maps form a commutative diagram with the natural embedding

$$
\mathcal{N} \otimes_{k} \mathcal{D}^{*} \otimes_{k} \mathfrak{P} \longrightarrow \mathcal{N} \otimes_{k} \operatorname{Hom}_{k}(\mathcal{D}, \mathfrak{P})
$$

To prove part (a), one considers the induced map of the dual vector spaces

$$
\left(\mathcal{N} \square_{\mathcal{D}} \mathfrak{P}\right)^{*} \simeq \operatorname{Hom}^{\mathcal{D}}\left(\mathfrak{P}, \mathcal{N}^{*}\right) \longrightarrow \operatorname{Hom}_{\mathcal{D}^{*}}\left(\mathfrak{P}, \mathcal{N}^{*}\right) \simeq\left(\mathcal{N} \otimes_{\mathcal{D}^{*}} \mathfrak{P}\right)^{*}
$$

and applies Proposition 2.9.

To prove part (b), notice that any right $\mathcal{D}$-comodule $\mathcal{N}$ is the union of its maximal $\mathcal{E}$-subcomodules $\mathcal{N}_{\mathcal{E}}$ over all the finite-dimensional subcoalgebras $\mathcal{E} \subset \mathcal{D}$. Since both the tensor and the contratensor products preserve inductive limits in their first arguments, it suffices to consider the case of a right $\mathcal{E}$-comodule $\mathcal{N}=\mathcal{N}_{\mathcal{E}}$. Then one has $\mathcal{N} \otimes_{\mathcal{D}^{*}} \mathfrak{P} \simeq \mathcal{N} \otimes_{\mathcal{E}^{*}}\left(\mathcal{E}^{*} \otimes_{\mathcal{D}^{*}} \mathfrak{P}\right)$ and $\mathcal{N} \odot_{\mathcal{D}} \mathfrak{P} \simeq \mathcal{N} \otimes_{\mathcal{E}^{*}}{ }^{\varepsilon} \mathfrak{P}$, so it remains to show that the natural map

$$
\mathcal{E}^{*} \otimes_{\mathcal{D}^{*}} \mathfrak{P} \longrightarrow{ }^{\varepsilon} \mathfrak{P} \simeq \mathcal{E}^{*} \odot_{\mathcal{D}} \mathfrak{P} \simeq \operatorname{Cohom}_{\mathcal{D}}(\mathcal{E}, \mathfrak{P})
$$

is an isomorphism. For this purpose, one presents the left $\mathcal{D}$-comodule $\mathcal{E}$ as the kernel of a morphism of finitely cogenerated cofree left $\mathcal{D}$-comodules and uses the right exactness property of the functor $\operatorname{Cohom}_{\mathcal{D}}$ together with the natural isomorphism

$$
\mathcal{J}^{*} \otimes_{\mathcal{D}^{*}} \mathfrak{P}=\left(V^{*} \otimes_{k} \mathcal{D}^{*}\right) \otimes_{\mathcal{D}^{*}} \mathfrak{P} \simeq \operatorname{Cohom}_{\mathcal{D}}\left(\mathcal{D} \otimes_{k} V, \mathfrak{P}\right)=\operatorname{Cohom}_{\mathcal{D}}(\mathcal{J}, \mathfrak{P})
$$

for a finitely cogenerated cofree left $\mathcal{D}$-comodule $\mathcal{J}=\mathcal{D} \otimes_{k} V$ and any left $\mathcal{D}$-contramodule $\mathfrak{P}$.

\section{MGM Duality for Coalgebras}

We start with several constructions and lemmas related to complexes of comodules and contramodules. These are purported to clear way to our key definition of a dedualizing complex of bicomodules over a pair of cocoherent coalgebras.

Let $\mathcal{C}$ and $\mathcal{D}$ be two coassociative coalgebras (with counits) over the same field $k$. Given a derived category symbol $\star=b,+,-, \varnothing$, abs + , abs - , or abs, we denote by $\mathrm{D}^{\star}(\mathcal{C}$-comod $)$ and $\mathrm{D}^{\star}(\mathcal{D}$-contra $)$ the corresponding (conventional or absolute) derived categories of the abelian categories $\mathcal{C}$-comod and $\mathcal{D}$-contra of left $\mathcal{C}$-comodules and left $\mathcal{D}$-contramodules (see [27, Appendix A] or [29, Appendix A] for the definitions). 
For any subcoalgebra $\mathcal{E}$ in a coalgebra $\mathcal{C}$, the maximal $\mathcal{E}$-subcomodule functor $\mathcal{M} \longmapsto \varepsilon_{\varepsilon} \mathcal{M}$ acting from the category of left $\mathcal{C}$-comodules to the category of left $\mathcal{E}$-comodules is left exact. The abelian category $\mathcal{C}$-comod has enough injective objects, which are precisely the direct summands of cofree $\mathcal{C}$-comodules. So one can identify the bounded below derived category $\mathrm{D}^{+}(\mathcal{C}$-comod) with the homotopy category of injective $\mathcal{C}$-comodules $\operatorname{Hot}^{+}\left(\mathcal{C}-\right.$ comodinj $\left._{\text {inj }}\right)$ and, applying the functor $\mathcal{M} \longmapsto \varepsilon \mathcal{M}$ to complexes of injective $\mathcal{C}$-comodules termwise, obtain the right derived functor

$$
\mathcal{M} \bullet \longmapsto{ }_{\varepsilon}^{\mathbb{R}} \mathcal{M} \bullet: \mathrm{D}^{+}(\mathcal{C}-\text { comod }) \longrightarrow \mathrm{D}^{+}(\mathcal{E} \text {-comod }) \text {. }
$$

Lemma 3.1. Let $\mathcal{C}$ be a left co-Noetherian coalgebra and $\mathcal{E} \subset \mathcal{C}$ be a subcoalgebra such that the quotient coalgebra without counit $\mathrm{C} / \mathcal{E}$ is conilpotent. Then a complex $\mathcal{L}^{\bullet} \in \mathrm{D}^{+}(\mathrm{C}$-comod $)$ has finitely cogenerated $\mathcal{C}$-comodules of cohomology if and only if the complex ${ }_{\mathcal{E}}^{\mathbb{R}} \mathcal{L} \cdot \in \mathrm{D}^{+}(\mathcal{E}$-comod) has finitely cogenerated $\mathcal{E}$-comodules of cohomology.

Proof. Notice that the cohomology $\mathcal{E}$-comodules of the complex ${ }_{\mathcal{E}}^{\mathbb{R}} \mathcal{L}$ are finitely cogenerated for any finitely cogenerated left $\mathcal{C}$-comodule $\mathcal{L}$ (viewed as a one-term complex of left $\mathcal{C}$-comodules). Indeed, one can compute the derived category object $\frac{\mathbb{R}}{\mathcal{L}}$ using a right resolution of the $\mathcal{C}$-comodule $\mathcal{L}$ by finitely cogenerated cofree left $\mathcal{C}$-comodules (which exists since the class of finitely cogenerated left comodules over a left coNoetherian coalgebra $\mathcal{C}$ is closed under the passages to the cokernels of morphisms) and apply Lemma 2.2(a). Since the class of finitely cogenerated left $\mathcal{E}$-comodules is also closed under the kernels, cokernels, and extensions, the desired assertion now follows by induction in the cohomological degree from Lemma 2.2(d).

We recall from Section 2 that finitely copresented left comodules over a left cocoherent coalgebra $\mathcal{C}$ form an abelian category. Notice that this abelian category has enough injective objects, which are precisely the direct summands of finitely cogenerated cofree $\mathcal{C}$-comodules.

Lemma 3.2. Let $\mathcal{C}$ be a left cocoherent coalgebra, and let $\mathcal{L} \bullet$ be a bounded below complex of left $\mathcal{C}$-comodules with finitely copresented cohomology modules. Then there exists a bounded below complex of finitely cogenerated cofree left $\mathcal{C}$-comodules $\boldsymbol{f} \bullet$ together with a quasi-isomorphism of complexes of left $\mathcal{C}$-comodules $\mathcal{L} \bullet \longrightarrow$

Proof. This is a standard step-by-step construction (cf. [30, Lemma 1.2] or the proof of $[29$, Lemma B.1(c)]).

A finite complex of left $\mathcal{C}$-comodules $\mathcal{L}^{\bullet}$ is said to have projective dimension $\leqslant d$ if one has $\operatorname{Hom}_{\mathrm{D}^{\mathrm{b}}(\mathcal{C}-\text { comod })}(\mathcal{L} \bullet, \mathcal{M}[n])=0$ for all left $\mathcal{C}$-comodules $\mathcal{M}$ and all the integers $n>d$. Similarly, a finite complex of left $\mathcal{D}$-contramodules $\mathfrak{Q} \bullet$ is said to have injective dimension $\leqslant d$ if one has $\operatorname{Hom}_{\mathrm{D}^{\mathrm{b}}(\mathcal{D} \text {-contra) }}\left(\mathfrak{P}, \mathfrak{Q}^{\bullet}[n]\right)=0$ for all left $\mathcal{D}$-contramodules $\mathfrak{P}$ and all $n>d$.

The bounded above derived category $\mathrm{D}^{-}(\mathcal{D}$-contra $)$ is equivalent to the homotopy category $\mathrm{Hot}^{-}\left(\mathcal{D}-\right.$ contra $\left.{ }_{\text {proj }}\right)$ of bounded above complexes of projective left $\mathcal{D}$-contramodules. Given a complex of right $\mathcal{D}$-comodules $\mathcal{N} \bullet$ and a bounded above complex of left $\mathcal{D}$-contramodules $\mathfrak{P}^{\bullet}$, we denote by $\operatorname{Ctrtor}_{*}^{\mathcal{D}}\left(\mathcal{N}^{\bullet}, \mathfrak{P}^{\bullet}\right)$ the homology 
vector spaces

$$
\operatorname{Ctrtor}_{n}^{\mathcal{D}}\left(\mathcal{N}^{\bullet}, \mathfrak{P}^{\bullet}\right)=H^{-n}\left(\mathcal{N}^{\bullet} \odot_{\mathcal{D}} \mathfrak{F}^{\bullet}\right)
$$

of the contratensor product of the complex $\mathcal{N} \bullet$ with a bounded above complex of projective left $\mathcal{D}$-contramodules $\mathfrak{F}^{\bullet}$ quasi-isomorphic to the complex $\mathfrak{P} \bullet$.

Here the bicomplex $\mathcal{N}^{\bullet} \odot_{\mathcal{D}} \mathfrak{F}^{\bullet}$ is presumed to be totalized by taking infinite direct sums along the diagonals. For any complex of right $\mathcal{D}$-comodules $\mathcal{N} \bullet$, any bounded above complex of left $\mathcal{D}$-contramodules $\mathfrak{P}^{\bullet}$, and any $k$-vector space $V$ there are natural isomorphisms of $k$-vector spaces

$$
\operatorname{Hom}_{k}\left(\operatorname{Ctrtor}_{n}^{\mathcal{D}}\left(\mathcal{N}^{\bullet}, \mathfrak{P}^{\bullet}\right), V\right) \simeq \operatorname{Hom}_{\mathrm{D}(\mathcal{D}-\text { contra })}\left(\mathfrak{P}^{\bullet}, \operatorname{Hom}_{k}\left(\mathcal{N}^{\bullet}, V\right)[n]\right)
$$

A finite complex of right $\mathcal{D}$-comodules $\mathcal{N} \bullet$ is said to have contraflat dimension $\leqslant d$ if one has $\operatorname{Ctrtor}_{n}^{\mathcal{D}}(\mathcal{N} \bullet, \mathfrak{P})=0$ for all left $\mathcal{D}$-contramodules $\mathfrak{P}$ and all the integers $n>d$. The contraflat dimension of a finite complex of right $\mathcal{D}$-comodules $\mathcal{N}^{\bullet}$ is equal to the injective dimension of the finite complex of left $\mathcal{D}$-contramodules $\mathfrak{Q}^{\bullet}=\operatorname{Hom}_{k}\left(\mathcal{N}^{\bullet}, V\right)$ for any $k$-vector space $V \neq 0$.

Lemma 3.3. If the coalgebra $\mathcal{D}$ is right cocoherent and left co-Noetherian, then the contraflat dimension of any finite complex of right $\mathcal{D}$-comodules $\mathcal{N} \cdot$ does not exceed its projective dimension.

Proof. Let $d$ be the projective dimension of the complex of right $\mathcal{D}$-comodules $\mathcal{N} \bullet$. For any finitely copresented right $\mathcal{D}$-comodule $\mathcal{L}$ there are natural isomorphisms of complexes of vector spaces

$$
\operatorname{Hom}_{\mathcal{D}}(\mathcal{N} \bullet, \mathcal{L}) \simeq \operatorname{Hom}^{\mathcal{D}}\left(\mathcal{L}^{*}, \mathcal{N}^{\bullet *}\right) \simeq\left(\mathcal{N} \bullet \odot_{\mathcal{D}} \mathcal{L}^{*}\right)^{*}
$$

(see Proposition 2.9), implying natural isomorphisms of cohomology spaces

$$
\operatorname{Hom}_{\mathrm{D}^{\mathrm{b}}(\mathcal{D}-\text { comod })}\left(\mathcal{N}^{\bullet}, \mathcal{L}[n]\right) \simeq \operatorname{Hom}_{\mathrm{D}^{\mathrm{b}}(\mathcal{D} \text {-contra })}\left(\mathcal{L}^{*}, \mathcal{N}^{\bullet *}[n]\right) \simeq \operatorname{Ctrtor}_{n}^{\mathcal{D}}\left(\mathcal{N}^{\bullet}, \mathcal{L}^{*}\right)^{*}
$$

Since any finitely presented left $\mathcal{D}$-contramodule $\mathfrak{P}$ has the form $\mathcal{L}^{*}$ for a certain finitely copresented right $\mathcal{D}$-contramodule $\mathcal{L}$, it follows that the supremum of all integers $n$ for which there exists a finitely presented left $\mathcal{D}$-contramodule $\mathfrak{P}$ with $\operatorname{Ctrtor}_{n}^{\mathcal{D}}(\mathcal{N} \bullet, \mathfrak{P}) \neq 0$ does not exceed $d$.

Furthermore, by Lemma 2.13(b) the functor of contratensor product $\odot_{\mathcal{D}}$ is isomorphic to the tensor product functor $\otimes_{\mathcal{D}^{*}}$ over the algebra $\mathcal{D}^{*}$ on the whole categories of arbitrary right $\mathcal{D}$-comodules and left $\mathcal{D}$-contramodules. Besides, the free $\mathcal{D}$-contramodules are the direct summands of infinite products of copies of the $\mathcal{D}$-contramodule $\mathcal{D}^{*}$. Since the coalgebra $\mathcal{D}$ is left (co-Noetherian and consequently) cocoherent, the algebra $\mathcal{D}^{*}$ is right coherent, so infinite products of flat left $\mathcal{D}^{*}$-modules are flat. In particular, projective left $\mathcal{D}$-contramodules are flat as left modules over $\mathcal{D}^{*}$. It follows that the functor $\mathrm{Ctrtor}^{\mathcal{D}}$ is isomorphic to the derived functor $\operatorname{Tor}^{\mathcal{D}^{*}}$ of tensor product of (complexes of) $\mathcal{D}^{*}$-modules on the whole domain of definition of the former derived functor.

Finally, since the coalgebra $\mathcal{D}$ is left cocoherent, the algebra $\mathcal{D}^{*}$ is right coherent and the abelian category of finitely presented left $\mathcal{D}^{*}$-modules is isomorphic to the abelian category of finitely presented left $\mathcal{D}$-contramodules. Any left $\mathcal{D}^{*}$-module is a 
filtered inductive limit of finitely presented ones, and the functor of tensor product over $\mathcal{D}^{*}$ preserves filtered inductive limits. The homological dimension of the functor $\operatorname{Tor}_{*}^{\mathcal{D}}\left(\mathcal{N}^{\bullet},-\right)$ on the abelian category of finitely presented left $\mathcal{D}^{*}$-modules does not exceed $d$, hence the homological dimension of this derived functor on the abelian category of arbitrary left $\mathcal{D}^{*}$-modules does not exceed $d$, either.

Now we finally come to the main definition of this section. Assume that the coalgebra $\mathcal{C}$ is left cocoherent and the coalgebra $\mathcal{D}$ is right cocoherent. A finite complex of $\mathcal{C}$-D-bicomodules $\mathcal{B}^{\bullet}$ is called a dedualizing complex for the pair of coalgebras $\mathcal{C}$ and $\mathcal{D}$ if the following conditions hold:

(i) the complex $\mathcal{B}^{\bullet}$ has finite projective dimension as a complex of left $\mathcal{C}$-comodules and finite contraflat dimension a complex of right $\mathcal{D}$-comodules;

(ii) the homothety maps $\mathcal{C}^{*} \longrightarrow \operatorname{Hom}_{\mathrm{D}^{\mathrm{b}}(\operatorname{comod}-\mathcal{D})}\left(\mathcal{B}^{\bullet}, \mathcal{B}^{\bullet}[*]\right)$ and $\mathcal{D}^{* \mathrm{op}} \longrightarrow$ $\operatorname{Hom}_{\mathrm{D}^{\mathrm{b}}(\mathcal{C}-\text {-comod })}\left(\mathcal{B}^{\bullet}, \mathcal{B}^{\bullet}[*]\right)$ are isomorphisms of graded rings; and

(iii) the bicomodules of cohomology of the complex $\mathcal{B}^{\bullet}$ are finitely copresented left $\mathcal{C}$-comodules and finitely copresented right $\mathcal{D}$-comodules.

Here the notation comod- $\mathcal{D}$ stands for the abelian category of right $\mathcal{D}$-comodules, and $\mathrm{D}^{\mathrm{b}}(\operatorname{comod}-\mathcal{D})$ is its bounded derived category. The dedualizing complex $\mathcal{B}^{\bullet}$ itself is viewed as an object of the bounded derived category $\mathrm{D}^{\mathrm{b}}(\mathcal{C}$-comod- $\mathcal{D})$ of the abelian category $\mathcal{C}$-comod- $\mathcal{D}$ of $\mathcal{C}$ - $\mathcal{D}$-bicomodules.

The homothety maps are induced by the left action of the algebra $\mathcal{C}^{*}$ by right $\mathcal{D}$-comodule endomorphisms of (every term of) the complex $\mathcal{B}^{\bullet}$ and the right action of the algebra $\mathcal{D}^{*}$ by left $\mathcal{C}$-comodule endomorphisms of $\mathcal{B} \bullet$.

We refer to the paper [30] and the references therein for a discussion of the classical notion of a dualizing complex over a pair of noncommutative rings, after which the above definition is largely modelled. A discussion of bicomodules can be found in [28, Section 2.6] and the references therein.

Example 3.4. For any coassociative coalgebra $\mathcal{C}$, the homological dimensions of the abelian categories of left $\mathcal{C}$-comodules, right $\mathcal{C}$-comodules, and left $\mathcal{C}$-contramodules coincide (see [25, Section 4.5], cf. [26, Corollary 1.9.4]). The common value of these three numbers (or infinity) is called the homological dimension of a coalgebra $\mathcal{C}$.

Let $\mathcal{C}$ be a left and right cocoherent coalgebra of finite homological dimension. For example, the coalgebra dual to the algebra of quantum formal power series from Examples 2.12 satisfies these assumptions. Then the one-term complex $\mathcal{B}^{\bullet}=\mathcal{C}$ is a dedualizing complex for the pair of coalgebras $(\mathcal{C}, \mathcal{C})$, as the conditions (i-iii) are obviously true for $\mathcal{B}^{\bullet}$.

More generally, a coalgebra $\mathcal{C}$ is called left Gorenstein if it has finite projective dimension as a left $\mathcal{C}$-comodule and finite contraflat dimension as a right $\mathcal{C}$-comodule. (The second condition can be rephrased by saying that the injective dimension of the left $\mathcal{C}$-contramodule $\mathcal{C}^{*}$ is finite.) For any left and right cocoherent, left Gorenstein coalgebra $\mathcal{C}$, the one-term complex $\mathcal{B}^{\bullet}=\mathcal{C}$ is a dedualizing complex for the pair of coalgebras $(\mathcal{C}, \mathcal{C})$. 
Example 3.5. Let $R$ be a finitely generated commutative algebra over a field $k$ and $I \subset R$ be a maximal ideal. Then the quotient algebras $R / I^{n}$ are finite-dimensional, so their dual vector spaces are cocommutative coalgebras over $k$, as is their inductive limit $\mathcal{C}=\lim _{n}\left(R / I^{n}\right)^{*}$. According to Examples 2.12 or Lemma 2.10(b), this coalgebra is Artinian, and consequently, by Lemma 2.3(a), co-Noetherian and cocoherent. The category of $\mathcal{C}$-comodules is isomorphic to the category of $I$-torsion $R$-modules in the sense of $[29$, Section 1$], \mathcal{C}-$ comod $\simeq R-\bmod _{I \text {-tors }}$. Moreover, the category of $\mathcal{C}$-contramodules is isomorphic to the category of $I$-contramodule $R$-modules as defined in [29, Section 2], $\mathcal{C}-$ contra $\simeq R-\bmod _{I \text {-ctra }}$ (see [28, Sections $\left.2.1-2.3\right]$ ).

Of course, the coalgebra $\mathcal{C}$ is cocommutative. A complex of $\mathcal{C}$-comodules $\mathcal{B}^{\bullet}$ is a dedualizing complex for the pair of coalgebras $(\mathcal{C}, \mathcal{C})$ in the sense of the above definition if and only if it is a dedualizing complex of $I$-torsion $R$-modules in the sense of the definition in [29, Section 4]. Indeed, the two conditions (i) are equivalent by Lemma 3.3 ; the two conditions (ii) are equivalent because $\mathfrak{R}=\mathfrak{C}^{*}$, and the two conditions (iii) are equivalent since, the coalgebra $\mathcal{C}$ being Artinian, a $\mathcal{C}$-comodule is finitely copresented if and only if it is Artinian. In particular, the dedualizing complex of $I$-torsion $R$-modules constructed in [29, Example 4.8] provides an example of a dedualizing complex of $\mathcal{C}$ - - -bicomodules.

For any $\mathcal{C}$-D $\mathcal{D}$-bicomodule $\mathcal{K}$ and any left $\mathcal{C}$-comodule $\mathcal{M}$, the $k$-vector space $\operatorname{Hom}_{\mathcal{C}}(\mathcal{K}, \mathcal{M})$ is endowed with the left $\mathcal{D}$-contramodule structure of a subcontramodule of the $\mathcal{D}$-contramodule $\operatorname{Hom}_{k}(\mathcal{K}, \mathcal{M})$. Similarly, for any $\mathcal{C}-\mathcal{D}$-bicomodule $\mathcal{K}$ and any left $\mathcal{D}$-contramodule $\mathfrak{P}$, the contratensor product $\mathcal{K} \odot_{\mathcal{D}} \mathfrak{P}$ is endowed with the left $\mathcal{C}$-comodule structure of a quotient comodule of the left $\mathcal{C}$-comodule $\mathcal{K} \otimes_{k} \mathfrak{P}$. For any $\mathcal{C}$-D $\mathcal{D}$-bicomodule $\mathcal{K}$, any left $\mathcal{C}$-comodule $\mathcal{N}$, and any left $\mathcal{D}$-contramodule $\mathfrak{P}$, there is a natural adjunction isomorphism of $k$-vector spaces [28, Section 3.1]

$$
\operatorname{Hom}_{\mathcal{C}}\left(\mathcal{K} \odot_{\mathcal{D}} \mathfrak{P}, \mathcal{M}\right) \simeq \operatorname{Hom}^{\mathcal{D}}\left(\mathfrak{P}, \operatorname{Hom}_{\mathcal{C}}(\mathcal{K}, \mathcal{M})\right) .
$$

The following theorem is the main result of this paper.

Theorem 3.6. Given a dedualizing complex $\mathcal{B} \bullet$ for a left cocoherent coalgebra $\mathcal{C}$ and a right cocoherent coalgebra $\mathcal{D}$ over a field $k$, for any symbol $\star=\mathrm{b},+,-, \varnothing$, abs + , abs-, or abs there is an equivalence of derived categories (2)

$$
\mathrm{D}^{\star}(\mathcal{C} \text {-comod }) \simeq \mathrm{D}^{\star}(\mathcal{D} \text {-contra })
$$

provided by mutually inverse functors $\mathbb{R} \operatorname{Hom}_{\mathcal{C}}(\mathcal{B} \bullet,-)$ and $\mathcal{B} \bullet \odot_{\mathcal{D}}^{\mathbb{L}}-$.

Proof. (Cf. the proofs of [29, Theorems 4.9 and 5.10].) Assume for simplicity of notation that the complex $\mathcal{B}^{\bullet}$ is concentrated in nonpositive cohomological degrees. Let $d$ be an integer greater or equal to both the projective dimension of the complex $\mathcal{B}^{\bullet}$ viewed as a complex of left $\mathcal{C}$-comodules and the contraflat dimension of $\mathcal{B}^{\bullet}$ as a complex of right $\mathcal{D}$-comodules.

To construct the image of a complex of left $\mathcal{C}$-comodules $\mathcal{M} \bullet$ under the functor $\mathbb{R} \operatorname{Hom}_{\mathcal{C}}\left(\mathcal{B}^{\bullet},-\right)$, one has to choose an exact sequence of complexes of left $\mathcal{C}$-comodules $0 \longrightarrow \mathcal{N}^{\bullet} \longrightarrow \mathcal{J}^{0} \bullet \longrightarrow \mathcal{J}^{1, \bullet} \longrightarrow \cdots$ with injective left $\mathcal{C}$-comodules $\mathrm{J}^{j, i}$. Then one applies the functor $\operatorname{Hom}_{\mathcal{C}}\left(\mathcal{B}^{\bullet},-\right)$ to every complex $0 \longrightarrow \mathrm{J}^{0, i} \longrightarrow \mathrm{J}^{1, i} \longrightarrow \mathrm{J}^{2, i} \longrightarrow \cdots$, 
obtaining a nonnegatively graded complex of left $\mathcal{D}$-contramodules $0 \longrightarrow \mathfrak{P}^{0, i} \longrightarrow$ $\mathfrak{P}^{1, i} \longrightarrow \mathfrak{P}^{2, i} \longrightarrow \cdots$. According to the projective dimension condition on the dedualizing complex $\mathcal{B}^{\bullet}$, the complex $\mathfrak{P}^{\bullet}, i$ has zero cohomology contramodules at the cohomological degrees above $d$; so it is quasi-isomorphic to its canonical truncation complex $\tau_{\leqslant d} \mathfrak{P}^{\bullet}, i$. By the definition, one sets the object $\mathbb{R} \operatorname{Hom}_{\mathcal{C}}\left(\mathcal{B}^{\bullet}, \mathcal{M}^{\bullet}\right)$ in the derived category $\mathrm{D}^{\star}(\mathcal{D}$-contra $)$ to be represented by the total complex of the bicomplex $\tau_{\leqslant d} \mathfrak{P}^{\bullet} \bullet$ concentrated in the cohomological degrees $0 \leqslant j \leqslant d$ and $i \in \mathbb{Z}$.

Similarly, to construct the image of a complex of left $\mathcal{D}$-contramodules $\mathfrak{P}^{\bullet}$ under the functor $\mathcal{B}^{\bullet} \odot{ }_{\mathcal{D}}^{\mathbb{L}}-$, one has to choose an exact sequence of complexes of left $\mathcal{D}$-contramodules $\cdots \longrightarrow \mathfrak{F}^{-1, \bullet} \longrightarrow \mathfrak{F}^{0} \bullet \longrightarrow \mathfrak{P}^{\bullet} \longrightarrow 0$ with projective left $\mathcal{D}$-contramodules $\mathfrak{F}^{j, i}$. Then one applies the functor $\mathcal{B}^{\bullet} \odot_{\mathcal{D}}-$ to every complex $\cdots \longrightarrow \mathfrak{F}^{-2, i} \longrightarrow \mathfrak{F}^{-1, i} \longrightarrow \mathfrak{F}^{0, i} \longrightarrow 0$, obtaining a nonpositively graded complex of left C-comodules $\cdots \longrightarrow \mathcal{M}^{-2, i} \longrightarrow \mathcal{M}^{-1, i} \longrightarrow \mathcal{M}^{0, i} \longrightarrow 0$. According to the contraflat dimension condition on the complex $\mathcal{B}^{\bullet}$, the complex $\mathcal{M}^{\bullet}, i$ has zero cohomology comodules at the cohomological degrees below $-d$; so it is quasi-isomorphic to its canonical truncation complex $\tau_{\geqslant-d} \mathcal{M} \mathfrak{M}^{\bullet}, i$. One sets the object $\mathcal{B}^{\bullet} \odot_{\mathcal{D}}^{\mathbb{L}} \mathfrak{P} \bullet$ in the derived category $\mathrm{D}^{\star}(\mathrm{C}$-comod $)$ to be represented by the total complex of the bicomplex $\tau_{\geqslant-d}(\mathcal{M} \cdot \bullet \cdot \bullet)$ concentrated in the cohomological degrees $-d \leqslant j \leqslant 0$ and $i \in \mathbb{Z}$.

These constructions of two derived functors are but particular cases of the construction of a derived functor of finite homological dimension spelled out in [29, Appendix B]. According to the results of that appendix, the above constructions produce well-defined triangulated functors $\mathbb{R} \operatorname{Hom}_{\mathcal{C}}\left(\mathcal{B}^{\bullet},-\right): \mathrm{D}^{\star}(\mathcal{C}$-comod $) \longrightarrow \mathrm{D}^{\star}(\mathcal{D}$-contra $)$ and $\mathcal{B} \bullet \odot_{\mathcal{D}}^{\mathbb{L}}-: D^{\star}(\mathcal{D}$-contra $) \longrightarrow D^{\star}(\mathcal{C}$-comod $)$ for any derived category symbol $\star=b$, ,,$+- \varnothing$, abs + , abs-, or abs. Moreover, the former functor is right adjoint to the latter one. All these assertions only depend on the first condition (i) in the definition of a dedualizing complex.

It remains to prove that the adjunction morphisms are isomorphisms. Since the total complexes of finite acyclic complexes of complexes are absolutely acyclic, in order to check that the morphism $\mathfrak{P}^{\bullet} \longrightarrow \mathbb{R} \operatorname{Hom}_{\mathcal{C}}\left(\mathcal{B}^{\bullet}, \mathcal{B}^{\bullet} \odot_{\mathcal{D}}^{\mathbb{L}} \mathfrak{P}^{\bullet}\right)$ is an isomorphism in the derived category $\mathrm{D}^{\star}(\mathcal{D}$-contra $)$ for all the $\star$-bounded complexes of left $\mathcal{D}$-contramodules $\mathfrak{P}^{\bullet}$ it suffices to consider the case of a one-term complex $\mathfrak{P}^{\bullet}=\mathfrak{P}$ corresponding to a single $\mathcal{D}$-contramodule $\mathfrak{P}$. Furthermore, since a morphism in $\mathrm{D}^{\mathrm{b}}\left(\mathcal{D}\right.$-contra) is an isomorphism whenever it is an isomorphism in $\mathrm{D}^{-}(\mathcal{D}$-contra $)$, one can view the one-term complex $\mathfrak{P}$ as an object of the bounded above derived category $\mathrm{D}^{-}(\mathcal{D}$-contra $)$ and replace it with a free $\mathcal{D}$-contramodule resolution $\mathfrak{F}^{\bullet}$ of the contramodule $\mathfrak{P}$. Applying the same totalization argument to the complex $\mathfrak{F} \bullet$, the question reduces to proving that the adjunction morphism $\mathfrak{F} \longrightarrow \mathbb{R} \operatorname{Hom}_{\mathcal{C}}\left(\mathcal{B}^{\bullet}, \mathcal{B}^{\bullet} \odot_{\mathcal{D}}^{\mathbb{L}} \mathfrak{F}\right)$ is an isomorphism in $\mathrm{D}^{\mathrm{b}}(\mathcal{D}$-contra) for any free left $\mathcal{D}$-contramodule $\mathfrak{F}$.

So let $V$ be a $k$-vector space and $\mathfrak{F}=\operatorname{Hom}_{k}(\mathcal{D}, V)$ be the free left $\mathcal{D}$-contramodule generated by $V$; then one has $\mathcal{B} \bullet \odot_{\mathcal{D}}^{\mathbb{L}} \mathfrak{F}=\mathcal{B} \bullet \odot_{\mathcal{D}} \mathfrak{F}=\mathcal{B} \bullet \otimes_{k} V$. By the condition (iii) together with Lemma 3.2, there exists a bounded below complex of finitely cogenerated cofree left $\mathcal{C}$-comodules $\mathcal{J} \bullet$ together with a quasi-isomorphism of complexes of 
left $\mathcal{C}$-comodules $\mathcal{B}^{\bullet} \longrightarrow \mathcal{J}^{\bullet}$. We have to check that the natural map

$$
\operatorname{Hom}_{k}(\mathcal{D}, V) \longrightarrow \operatorname{Hom}_{\mathcal{C}}\left(\mathcal{B}^{\bullet}, \jmath^{\bullet} \otimes_{k} V\right)
$$

is a quasi-isomorphism of complexes of left $\mathcal{D}$-contramodules.

The left-hand side is the projective limit of the vector $\operatorname{spaces} \operatorname{Hom}_{k}(\mathcal{E}, V)$ over all the finite-dimensional subcoalgebras $\mathcal{E} \subset \mathcal{D}$, while the right-hand side is the projective limit of the complexes of vector spaces $\operatorname{Hom}_{\mathcal{E}}\left(\varepsilon \mathcal{B} \mathcal{B}^{\bullet} \mathcal{J}^{\bullet} \otimes_{k} V\right)$. In particular, the map $\mathcal{D}^{*} \longrightarrow \operatorname{Hom}_{\mathcal{C}}\left(\mathcal{B}^{\bullet}, \mathcal{J}^{\bullet}\right)$ is a morphism of complexes of profinitedimensional topological vector spaces. Being a quasi-isomorphism of complexes of discrete/nontopological vector spaces (with the topologies forgotten) by the condition (ii), it is consequently also a quasi-isomorphism of complexes in the abelian category of profinite-dimensional topological vector spaces.

For any profinite-dimensional topological $k$-vector space $K$ and any discrete $k$-vector space $V$ one denotes by $K \otimes \widehat{ } V$ the projective limit

$$
K \otimes \widehat{V}=\lim _{U} K / U \otimes_{k} V
$$

taken over all the open subspaces $U \subset K$ [28, Sections 2.3-4]. Equivalently, one can set $W^{*} \otimes \widehat{ } V=\operatorname{Hom}_{k}(W, V)$ for any discrete $k$-vector spaces $W$ and $V$. Both the abelian categories of discrete and profinite-dimensional vector spaces being semisimple, the additive functor $\otimes \widehat{ }$ is exact. Now one has

$$
\lim _{\mathcal{E}} \operatorname{Hom}_{\mathcal{E}}\left({ }_{\varepsilon} \mathcal{B}^{\bullet}, \varepsilon_{\varepsilon} \mathcal{J}^{\bullet} \otimes_{k} V\right) \simeq \lim _{\mathcal{E}} \operatorname{Hom}_{\mathcal{E}}\left(\varepsilon_{\mathcal{B}} \mathcal{B}^{\bullet}, \varepsilon_{\varepsilon} \jmath^{\bullet}\right) \otimes^{\wedge} V,
$$

since the complex of $\mathcal{E}$-comodules $\varepsilon_{\mathcal{B}} \mathcal{B}^{\bullet}$ is finite, while the terms of the complex $\varepsilon_{\mathcal{E}} \bullet^{\bullet}$ are finite-dimensional cofree $\mathcal{E}$-comodules. Finally, the morphism of complexes in question is obtained by applying the exact functor $-\otimes^{\wedge} V$ to the quasi-isomorphism of complexes $\mathcal{D}^{*} \longrightarrow \operatorname{Hom}_{\mathcal{C}}\left(\mathcal{B}^{\bullet}, \mathcal{\partial}^{\bullet}\right)$.

Similarly, in order to prove that the adjunction morphism $\mathcal{B}^{\bullet} \odot_{\mathcal{D}}^{\mathbb{L}} \mathbb{R} \operatorname{Hom}_{\mathcal{C}}\left(\mathcal{B}^{\bullet}, \mathcal{M}^{\bullet}\right)$ $\longrightarrow \mathcal{M} \bullet$ is an isomorphism in the derived category $\mathrm{D}^{\star}(\mathcal{C}$-comod $)$ for any $\star$-bounded complex of left $\mathcal{C}$-comodules $\mathcal{M}^{\bullet}$, it suffices to check that this morphism is an isomorphism in $\mathrm{D}^{\mathrm{b}}(\mathcal{C}$-comod) for any cofree left $\mathcal{C}$-comodule $\mathcal{J}$ viewed as a one-term complex in $\mathrm{D}^{\mathrm{b}}\left(\mathcal{C}\right.$-comod). Let $\mathcal{J}=\mathcal{C} \otimes_{k} V$ be a cofree left $\mathcal{C}$-comodule generated by a $k$-vector space $V$; then one has $\mathbb{R} \operatorname{Hom}_{\mathcal{C}}\left(\mathcal{B}^{\bullet}, \mathcal{J}\right)=\operatorname{Hom}_{\mathcal{C}}\left(\mathcal{B}^{\bullet}, \mathcal{J}\right)=\operatorname{Hom}_{k}\left(\mathcal{B}^{\bullet}, V\right)$. Let $\mathcal{J} \bullet$ be a bounded below complex of finitely cogenerated cofree right $\mathcal{D}$-comodules endowed with a quasi-isomorphism of complexes of right $\mathcal{D}$-comodules $\mathcal{B}^{\bullet} \longrightarrow \mathcal{J}^{\bullet}$. Then $\operatorname{Hom}_{k}\left(\mathcal{J}^{\bullet}, V\right)$ is a bounded above complex of free left $\mathcal{D}$-contramodules quasiisomorphic to $\operatorname{Hom}_{k}(\mathcal{B} \bullet V)$. We have to show that the map

$$
\mathcal{B}^{\bullet} \odot_{\mathcal{D}} \operatorname{Hom}_{k}\left(\mathcal{g}^{\bullet}, V\right) \longrightarrow \mathcal{C} \otimes_{k} V
$$

induced by the left $\mathcal{C}$-coaction in $\mathcal{B}^{\bullet}$ is a quasi-isomorphism (of complexes of left C-comodules).

The functors on both sides of our map preserve infinite direct sums and inductive limits in the argument $V$, so it suffices to consider the case $V=k$. Passing to the dual vector spaces, we have to check that the map $\mathcal{C}^{*} \longrightarrow \operatorname{Hom}^{\mathcal{D}}\left(\mathcal{J}^{\bullet *}, \mathcal{B}^{\bullet *}\right)$ is a quasi-isomorphism. The latter map is the composition $\mathcal{C}^{*} \longrightarrow \operatorname{Hom}_{\mathcal{D}^{\text {op }}}\left(\mathcal{B}^{\bullet}, g^{\bullet}\right) \longrightarrow$ 
$\operatorname{Hom}^{\mathcal{D}}\left(\mathcal{J}^{\bullet *}, \mathcal{B}^{\bullet *}\right)$ of the homothety map of the condition (ii) and the map induced by the dualization functor $\mathcal{N} \longmapsto \mathcal{N}^{*}$. It remains to apply Proposition 2.9(b).

\section{MGM Duality for Semialgebras}

Let $\mathcal{C}$ be a coassociative coalgebra over a field $k$. Then the operation of cotensor product $\square_{\mathcal{e}}$ (as defined in the end of Section 2) provides the category of C-C-bicomodules $\mathrm{C}$-comod-C with an associative and unital tensor category structure. The $\mathcal{C}$ - $\mathrm{C}$-bicomodule $\mathcal{C}$ is the unit object. A (semiassociative and semiunital) semialgebra over $\mathcal{C}$ is an (associative and unital) algebra object in this tensor category. In other words, a semialgebra $\mathcal{S}$ over $\mathcal{C}$ is a $\mathcal{C}$ - $\mathcal{C}$-bicomodule endowed with $\mathcal{C}$-C-bicomodule morphisms of semiunit $\mathcal{C} \longrightarrow \mathcal{S}$ and semimultiplication $\mathcal{S} \square_{\mathcal{e}} \mathcal{S} \longrightarrow \mathcal{S}$ satisfying the conventional associativity and unitality axioms. We refer to [24, Sections 0.3.1-2 and 1.3.1] and [28, Sections 2.5-6] for further details.

The cotensor product operation also provides the category of left $\mathcal{C}$-comodules $\mathcal{C}$-comod with the structure of left module category over the tensor category $\mathrm{C}$-comod- $\mathrm{C}$ and the category of right $\mathrm{C}$-comodules comod- $\mathrm{C}$ with the structure of right module category over $\mathcal{C}$-comod- $\mathcal{C}$. Furthermore, the functor of cohomomorphisms Cohom $_{\mathcal{C}}$ (see Section 2) defined the structure of a right module category over $\mathcal{C}$-comod- $\mathcal{C}$ on the category opposite to the category of left $\mathcal{C}$-contramodules $\mathcal{C}$-contra ${ }^{\mathrm{op}}$. Given a semialgebra $\mathcal{S}$ over $\mathcal{C}$, one can consider module objects over the algebra object $\mathcal{S} \in \mathcal{C}$-comod- $\mathcal{C}$ in the module categories $\mathcal{C}$-comod, comod- $\mathrm{C}$, and $\mathrm{C}$-contra ${ }^{\text {op }}$ over the tensor category $\mathcal{C}$-comod- $\mathcal{C}$. This leads to the following definitions.

A left semimodule $\mathcal{M}$ over $\mathcal{S}$ is a left $\mathcal{C}$-comodule endowed with a left $\mathcal{C}$-comodule morphism of left semiaction $\mathcal{S} \square_{\mathcal{C}} \mathcal{M} \longrightarrow \mathcal{M}$ satisfying the associativity and unitality equations. A right semimodule $\mathcal{N}$ over $\mathcal{S}$ is a right $\mathcal{C}$-comodule endowed with a right $\mathcal{C}$-comodule morphism of right semiaction $\mathcal{N} \square_{\mathcal{e}} \mathcal{S} \longrightarrow \mathcal{N}$ satisfying the similar equations. Finally, a left semicontramodule $\mathfrak{P}$ over $\mathcal{S}$ is a left $\mathcal{C}$-contramodule endowed with a left $\mathcal{C}$-contramodule morphism of left semicontraaction $\mathfrak{P} \longrightarrow \operatorname{Cohom}_{\mathfrak{C}}(\mathfrak{S}, \mathfrak{P})$ satisfying the dual versions of the same equations. The details concerning semimodules can be found in the above references; and we refer to [24, Sections 0.3.4-5 and 3.3.1] and [28, Sections 2.5-6] for further details about semicontramodules.

The $k$-vector space of all morphisms $\mathcal{L} \longrightarrow \mathcal{M}$ in the category of left $\mathcal{S}$-semimodules $\mathcal{S}$-simod is denoted by $\operatorname{Hom}_{\mathcal{S}}(\mathcal{L}, \mathcal{M})$. Given a left $\mathcal{C}$-comodule $\mathcal{L}$, the left $\mathcal{S}$-semimodule $\mathcal{S} \square_{\mathcal{C}} \mathcal{L}$ is called the left $\mathcal{S}$-semimodule induced from the left $\mathcal{C}$-comodule $\mathcal{L}$. For any left $\mathcal{S}$-semimodule $\mathcal{M}$, there is a natural isomorphism of $k$-vector spaces

$$
\operatorname{Hom}_{\mathcal{S}}\left(\mathcal{S} \square_{\mathcal{C}} \mathcal{L}, \mathcal{M}\right) \simeq \operatorname{Hom}_{\mathcal{C}}(\mathcal{L}, \mathcal{M}) .
$$

The $k$-vector space of all morphisms $\mathfrak{P} \longrightarrow \mathfrak{Q}$ in the category of left $\mathcal{S}$-semicontramodules $\mathcal{S}$-sicntr is denoted by $\operatorname{Hom}^{\mathcal{S}}(\mathfrak{P}, \mathfrak{Q})$. For any right $\mathcal{S}$-semimodule $\mathcal{N}$ and $k$-vector space $V$, the left $\mathcal{C}$-contramodule $\operatorname{Hom}_{k}(\mathcal{N}, V)$ has a natural left 
$\mathcal{S}$-semicontramodule structure. Given a left $\mathcal{C}$-contramodule $\mathfrak{Q}$, the left $\mathcal{C}$-contramodule $\operatorname{Cohom}_{\mathfrak{C}}(\mathcal{S}, \mathfrak{Q})$ is endowed with a left $\mathcal{S}$-semicontramodule structure as a quotient semicontramodule of the left $\mathcal{S}$-semicontramodule $\operatorname{Hom}_{k}(\mathcal{S}, \mathfrak{Q})$. The left $\mathcal{S}$-semicontramodule $\operatorname{Cohom}_{\mathcal{L}}(\boldsymbol{S}, \mathfrak{Q})$ is called the left $\boldsymbol{S}$-semicontramodule coinduced from the left $\mathcal{C}$-contramodule $\mathfrak{Q}$. For any left $\mathcal{S}$-semicontramodule $\mathfrak{P}$, there is a natural isomorphism of $k$-vector spaces

$$
\operatorname{Hom}^{\mathcal{s}}\left(\mathfrak{P}, \operatorname{Cohom}_{\mathcal{C}}(\mathcal{S}, \mathfrak{Q})\right) \simeq \operatorname{Hom}^{\mathfrak{e}}(\mathfrak{P}, \mathfrak{Q}) .
$$

Our next aim is to define the operation of contratensor product $\mathcal{N} \odot s \mathfrak{P}$ of a right $\mathcal{S}$-semimodule $\mathcal{N}$ and a left $\mathcal{S}$-semicontramodule $\mathfrak{P}$ [24, Sections 0.3.7 and 6.1.1-2]. The idea is that $\mathcal{N} \odot s \mathfrak{P}$ is a $k$-vector space for which the natural isomorphism

$$
\operatorname{Hom}_{k}\left(\mathcal{N} \odot_{s} \mathfrak{P}, V\right) \simeq \operatorname{Hom}^{\boldsymbol{s}}\left(\mathfrak{P}, \operatorname{Hom}_{k}(\mathcal{N}, V)\right)
$$

holds for any $k$-vector space $V$. This condition determines the $k$-vector space $\mathcal{N} \odot s \mathfrak{P}$ uniquely up to a natural isomorphism. The following explicit construction shows that such a vector space exists.

The contratensor product $\mathcal{N} \odot s \mathfrak{P}$ is the cokernel of (the difference of) the pair of natural $k$-linear maps

$$
\left(\mathcal{N} \square_{e} \mathcal{S}\right) \odot_{e} \mathfrak{P} \rightrightarrows \mathcal{N} \odot_{e} \mathfrak{P}
$$

Here the first map is induced by the right $\mathcal{S}$-semiaction morphism $\mathcal{N} \square_{\mathfrak{e}} \mathcal{S} \longrightarrow \mathcal{N}$, while the second map is the composition of the left $\mathcal{S}$-semicontraaction morphism $\mathfrak{P} \longrightarrow \operatorname{Cohom}_{\mathfrak{C}}(\boldsymbol{S}, \mathfrak{P})$ and the natural "evaluation" map

$$
\eta_{\mathcal{S}}:\left(\mathcal{N} \square_{\mathcal{C}} \mathcal{S}\right) \odot_{\mathcal{e}} \operatorname{Cohom}_{\mathcal{C}}(\mathcal{S}, \mathfrak{P}) \longrightarrow \mathcal{N} \odot_{\mathcal{e}} \mathfrak{P}
$$

The "evaluation" map is defined for any two coalgebras $\mathcal{C}$ and $\mathcal{D}$ over $k$, a $\mathcal{C}$ - $\mathcal{D}$-bicomodule $\mathcal{K}$, a right $\mathcal{C}$-comodule $\mathcal{N}$, and a left $\mathcal{C}$-contramodule $\mathfrak{P}$,

$$
\eta_{\mathcal{K}}:\left(\mathcal{N} \square_{\mathcal{C}} \mathcal{K}\right) \odot_{\mathcal{D}} \operatorname{Cohom}_{\mathfrak{C}}(\mathcal{K}, \mathfrak{P}) \longrightarrow \mathcal{N} \odot_{\mathcal{e}} \mathfrak{P} \text {, }
$$

and can be characterized by the condition that the dual map $\eta_{K}^{*}=\operatorname{Hom}_{k}\left(\eta_{\mathcal{K}}, k\right)$ is equal to the map

$$
\operatorname{Hom}^{\mathfrak{e}}\left(\mathfrak{P}, \mathcal{N}^{*}\right) \longrightarrow \operatorname{Hom}^{\mathcal{D}}\left(\operatorname{Cohom}_{\mathcal{C}}(\mathcal{K}, \mathfrak{P}), \operatorname{Cohom}_{\mathcal{C}}\left(\mathcal{K}, \mathcal{N}^{*}\right)\right)
$$

provided by the functor $\operatorname{Cohom}_{\mathcal{C}}(\mathcal{K},-)$ : $\mathcal{C}$-contra $\longrightarrow \mathcal{D}$-contra. Even more explicitly, the $k$-linear map $\eta_{\mathcal{K}}$ is constructed as the unique map forming a commutative square with the composition of maps

$$
\left(\mathcal{N} \square_{\mathcal{e}} \mathcal{K}\right) \otimes_{k} \operatorname{Hom}_{k}(\mathcal{K}, \mathfrak{P}) \longrightarrow \mathcal{N} \otimes_{k} \mathcal{K} \otimes_{k} \operatorname{Hom}_{k}(\mathcal{K}, \mathfrak{P}) \longrightarrow \mathcal{N} \otimes_{k} \mathfrak{P}
$$

and the natural surjections. We refer to [24, Section 6.1.1] for further details.

For any right $\mathcal{C}$-comodule $\mathcal{N}$ and left $\mathcal{S}$-semicontramodule $\mathfrak{P}$, there is a natural isomorphism of $k$-vector spaces [24, Section 6.1.2]

$$
\left(\mathcal{N} \square_{\mathcal{e}} \mathcal{S}\right) \odot_{\mathcal{S}} \mathfrak{P} \simeq \mathcal{N} \odot_{\mathrm{e}} \mathfrak{P}
$$

The category $\mathcal{S}$-simod of left $\mathcal{S}$-semimodules is abelian provided that $\mathcal{S}$ is an injective right $\mathcal{C}$-comodule. In fact, $\boldsymbol{S}$ is an injective right $\mathcal{C}$-comodule if and only if the category $\mathcal{S}$-simod is abelian and the forgetful functor $\mathcal{S}$-simod $\longrightarrow \mathcal{C}$-comod is exact. 
Similarly, the category $\mathcal{S}$-sicntr of left $\mathcal{S}$-semicontramodules is abelian provided that $\mathcal{S}$ is an injective left $\mathcal{C}$-comodule. In fact, $\mathcal{S}$ is an injective left $\mathcal{C}$-comodule if and only if the category $\mathcal{S}$-sicntr is abelian and the forgetful functor $\mathcal{S}$-sicntr $\longrightarrow \mathcal{C}$-contra is exact. (See [28, Proposition 2.5] for a proof of the dual versions of these results.)

Let $\mathcal{S}$ be a semialgebra over a coalgebra $\mathcal{C}$ over $k$ and $\mathcal{T}$ be a semialgebra over a coalgebra $\mathcal{D}$ over $k$. An $\mathcal{S}-\mathcal{T}$-bisemimodule $\mathcal{K}$ is a $\mathcal{C}$-D -bicomodule endowed with a left $\mathcal{S}$-semimodule and a right $\mathcal{T}$-semimodule structures such that the semiaction maps $\mathcal{S} \square_{\mathcal{C}} \mathcal{K} \longrightarrow \mathcal{K}$ and $\mathcal{K} \square_{\mathcal{D}} \mathcal{T} \longrightarrow \mathcal{K}$ are morphisms of $\mathcal{C}$-D $\mathcal{D}$-bicomodules which commute with each other in the sense that the two compositions $\mathcal{S} \square_{\mathcal{C}} \mathcal{K} \square_{\mathcal{D}} \mathcal{T} \longrightarrow$ $\mathcal{K} \square_{\mathcal{D}} \mathcal{T} \longrightarrow \mathcal{K}$ and $\mathcal{S} \square_{\mathcal{C}} \mathcal{K} \square_{\mathcal{D}} \mathcal{T} \longrightarrow \mathcal{S} \square_{\mathcal{C}} \mathcal{K} \longrightarrow \mathcal{K}$ coincide. Alternatively, one can define an $\mathcal{S}$ - $\mathcal{T}$-bisemimodule as a $\mathcal{C}$ - $\mathcal{D}$-bicomodule endowed with a bisemiaction map $\mathcal{S} \square_{\mathcal{C}} \mathcal{K} \square_{\mathcal{D}} \mathcal{T} \longrightarrow \mathcal{K}$, which must be a morphism of $\mathcal{C}$ - $\mathcal{D}$-bicomodules satisfying the associativity and unitality axioms.

Let $\mathcal{K}$ be an $\mathcal{S}$ - $\mathcal{T}$-bisemimodule and $\mathfrak{P}$ be a left $\mathcal{T}$-semicontramodule. Assuming that $\mathcal{S}$ is an injective right $\mathcal{C}$-comodule, the contratensor product $\mathcal{K} \odot_{\mathcal{T}} \mathfrak{P}$ then has a natural left $\mathcal{S}$-semimodule structure. Similarly, let $\mathcal{K}$ be an $\mathcal{S}$ - $\mathcal{T}$-bisemimodule and $\mathcal{M}$ be a left $\mathcal{S}$-semimodule. Assuming that $\mathcal{T}$ is an injective left $\mathcal{D}$-comodule, the $k$-vector space of left $\mathcal{S}$-semimodule morphisms $\operatorname{Hom}_{\mathcal{S}}(\mathcal{K}, \mathcal{M})$ has a natural left $\mathcal{T}$-semicontramodule structure [24, Section 6.1.3].

Whenever $\mathcal{S}$ is an injective right $\mathcal{C}$-comodule and $\mathcal{T}$ is an injective left $\mathcal{D}$-comodule, for any $\mathcal{S}$ - $\mathcal{T}$-bisemimodule $\mathcal{K}$, any left $\mathcal{S}$-semimodule $\mathcal{M}$, and any right $\mathcal{T}$-semicontramodule $\mathfrak{P}$, there is a natural adjunction isomorphism of $k$-vector spaces [24, Section 6.1.4]

$$
\operatorname{Hom}_{\mathcal{S}}(\mathcal{K} \odot \mathcal{T} \mathfrak{P}, \mathcal{M}) \simeq \operatorname{Hom}^{\mathcal{T}}\left(\mathfrak{P}, \operatorname{Hom}_{\mathfrak{s}}(\mathcal{K}, \mathcal{M})\right)
$$

There are also some other situations in which there is a natural left $\mathcal{S}$-semimodule structure on the contratensor product $\mathcal{K} \odot \mathcal{T} \mathfrak{P}$ and a natural left $\mathcal{T}$-semicontramodule structure on the space of homomorphisms $\operatorname{Hom}_{\mathcal{S}}(\mathcal{K}, \mathcal{M})$. The case of $\mathcal{S}=\mathcal{K}=\mathfrak{T}$ is of particular interest. For any semialgebra $\mathcal{S}$ over a coalgebra $\mathcal{C}$, the functors $\Phi_{\mathcal{S}}: \mathfrak{P} \longmapsto \mathcal{S} \odot_{\mathcal{S}} \mathfrak{P}$ and $\Psi_{\mathcal{S}}: \mathcal{M} \longmapsto \operatorname{Hom}_{\mathcal{S}}(\mathcal{S}, \mathcal{M})$ establish an equivalence between the exact categories of $\mathcal{C}$-injective left $\mathcal{S}$-semimodules and $\mathcal{C}$-projective left $\mathcal{S}$-semicontramodules [24, Section 6.2]. This equivalence forms a commutative square with the forgetful functors and the equivalence between the additive categories of injective left $\mathcal{C}$-comodules and projective left $\mathcal{C}$-contramodules $\mathcal{C}$-comod -comj $_{\text {in }} \simeq$ $\mathcal{C}-$ contra $_{\text {proj }}$ provided by the functors $\Phi_{\mathcal{C}}=\mathcal{C} \odot_{\mathcal{C}}-$ and $\Psi_{\mathcal{C}}=\operatorname{Hom}_{\mathfrak{C}}(\mathcal{C},-)$.

Let $\mathcal{S}$ be a semialgebra over a coalgebra $\mathcal{C}$ over $k$ and $\mathcal{T}$ be a semialgebra over a coalgebra $\mathcal{D}$ over $k$. Assume that $\mathcal{S}$ is an injective right $\mathcal{C}$-comodule and $\mathcal{T}$ is an injective left $\mathcal{D}$-comodule. So the categories $\mathcal{S}$-simod and $\mathcal{T}$-sicntr are abelian.

Proposition 4.1. (a) There are enough injective objects in the abelian category $\boldsymbol{S}$-simod. A left $\mathcal{S}$-semimodule is injective if and only if it is a direct summand of a left $\mathcal{S}$-semimodule of the form $\Phi_{\boldsymbol{S}}\left(\operatorname{Hom}_{k}(\mathcal{S}, V)\right)$, where $V$ is a $k$-vector space. Furthermore, the forgetful functor $\mathcal{S}$-simod $\longrightarrow \mathcal{C}$-comod preserves injectives. 
(b) There are enough projective objects in the abelian category $\mathcal{T}$-sicntr. A left $\mathcal{T}$-semicontramodule is projective if and only if it is a direct summand of a left $\mathcal{T}$-semicontramodule of the form $\Psi_{\mathcal{T}}\left(\mathcal{T} \otimes_{k} V\right)$, where $V$ is a k-vector space. Furthermore, the forgetful functor $\mathcal{T}$-sicntr $\longrightarrow \mathcal{D}$-contra preserves projectives.

Proof. A proof of this result under slightly more restrictive assumptions (of a semialgebra injective over its coalgebra on both sides) can be found in [28, Proposition 3.5]. In the general case, there is an argument based on the results of [24, Section 6.2], proceeding as follows.

Part (a): the forgetful functor $\mathcal{S}$-simod $\longrightarrow \mathcal{C}$-comod preserves injectives, since it has an exact left adjoint functor $\mathcal{S} \square_{\mathcal{C}}$ - assigning to a left $\mathcal{C}$-comodule the induced left $\mathcal{S}$-semimodule. To prove that the left $\mathcal{S}$-semimodule $\mathcal{M}=\boldsymbol{S} \odot_{\mathcal{S}} \operatorname{Hom}_{k}(\boldsymbol{S}, V)$ is injective, one first notices that $\operatorname{Hom}_{k}(\boldsymbol{S}, V)$ is a projective left $\mathcal{C}$-contramodule, hence $\mathcal{M}$ is an injective left $\mathcal{C}$-comodule and $\operatorname{Hom}_{\mathcal{S}}(\mathcal{S}, \mathcal{M})=\Psi_{\mathcal{S}}(\mathcal{M}) \simeq \operatorname{Hom}_{k}(\mathcal{S}, V)$. Applying [24, Proposition 6.2.2(a)] for $\mathcal{T}=\mathcal{K}=\mathfrak{S}$, one computes that

$$
\operatorname{Hom}_{\mathcal{S}}(\mathcal{L}, \mathcal{M}) \simeq \operatorname{Hom}_{k}(\mathcal{L}, V)
$$

for any left $\mathcal{S}$-semimodule $\mathcal{L}$. This proves that $\mathcal{M}$ is injective; and in order to show that $\mathcal{L}$ can be embedded into a left $\mathcal{S}$-semimodule of the form $\Phi_{\mathcal{S}}\left(\operatorname{Hom}_{k}(\mathcal{S}, V)\right)$, it suffices to take $V=\mathcal{L}$.

Part (b): the forgetful functor $\mathcal{T}$-sicntr $\longrightarrow \mathcal{D}$-contra preserves projectives, since it has an exact right adjoint functor $\operatorname{Cohom}_{\mathcal{D}}(\mathcal{T},-)$ assigning to a left $\mathcal{D}$-contramodule

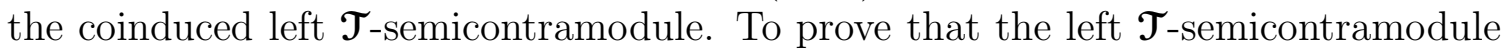
$\mathfrak{P}=\operatorname{Hom}_{\mathcal{T}}\left(\mathfrak{T}, \mathcal{T} \otimes_{k} V\right)$ is projective, one first notices that $\mathcal{T} \otimes_{k} V$ is an injective left $\mathcal{D}$-comodule, hence $\mathfrak{P}$ is a projective left $\mathcal{D}$-contramodule and $\mathcal{T} \odot_{\mathcal{T}} \mathfrak{P}=\Phi_{\mathcal{T}}(\mathfrak{P}) \simeq$ $\mathfrak{T} \otimes_{k} V$. Applying [24, Proposition 6.2.3(a)] for $\mathcal{S}=\mathcal{K}=\mathfrak{T}$, one computes that

$$
\operatorname{Hom}^{\mathfrak{T}}(\mathfrak{P}, \mathfrak{Q}) \simeq \operatorname{Hom}_{k}(V, \mathfrak{Q})
$$

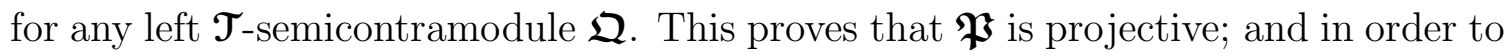

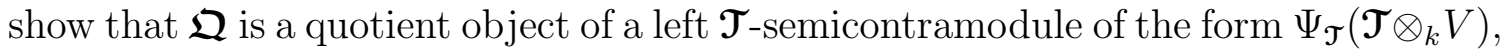
it suffices to take $V=\mathfrak{Q}$.

A finite complex of left $\mathcal{S}$-semimodules $\mathcal{L}^{\bullet}$ is said to have projective dimension $\leqslant d$ if one has $\operatorname{Hom}_{\mathrm{D}^{\mathrm{b}}(\mathcal{S} \text {-simod })}\left(\mathcal{L}^{\bullet}, \mathcal{M}[n]\right)=0$ for all left $\mathcal{S}_{\text {-semimodules }} \mathcal{M}$ and all the integers $n>d$. The projective dimension of the complex of left $\mathcal{S}$-semimodules $\mathcal{L}^{\bullet}=\mathcal{S} \square_{\mathcal{C}} \mathcal{L}^{\bullet}$ induced from a finite complex of left $\mathcal{C}$-comodules $\mathcal{L}^{\bullet}$ does not exceed the projective dimension of the complex of left $\mathcal{C}$-comodules $\mathcal{L} \bullet$.

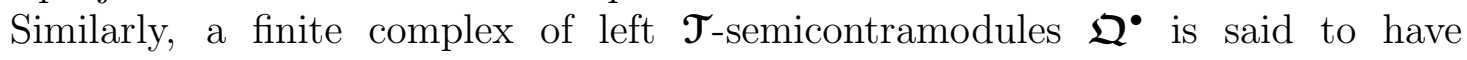

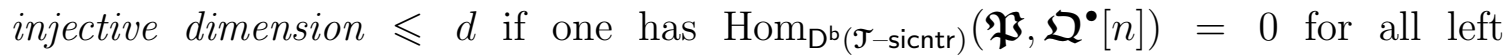
$\mathcal{T}$-semicontramodules $\mathfrak{P}$ and all $n>d$. The injective dimension of the complex of

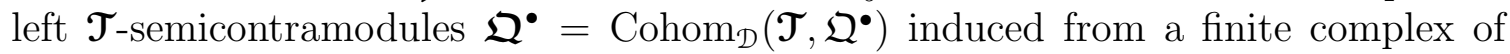
left $\mathcal{D}$-contramodules $\mathfrak{Q}^{\bullet}$ does not exceed the injective dimension of the complex of left $\mathcal{D}$-contramodules $\mathfrak{Q} \bullet$.

The bounded above derived category $\mathrm{D}^{-}(\mathcal{T}$-sicntr $)$ is equivalent to the homotopy category $\operatorname{Hot}^{-}(\mathcal{T}$-sicntr proj $)$ of bounded above complexes of projective left 
$\mathcal{T}$-semicontramodules. Given a complex of right $\mathcal{T}_{\text {-semimodules }} \mathcal{N}^{\bullet}$ and a bounded above complex of left $\mathcal{T}_{\text {-semicontramodules }} \mathfrak{P}^{\bullet}$, we denote by $\operatorname{Ctr}_{\operatorname{Tor}}^{\mathcal{T}}\left(\mathcal{N}^{\bullet}, \mathfrak{P}^{\bullet}\right)$ the homology vector spaces

$$
\operatorname{Ctr}_{\operatorname{Tor}}^{\mathfrak{T}}\left(\mathcal{N}^{\bullet}, \mathfrak{P}^{\bullet}\right)=H^{-n}\left(\mathcal{N}^{\bullet} \odot \mathcal{T} \mathfrak{F}^{\bullet}\right)
$$

of the contratensor product of the complex of right $\mathcal{T}_{\text {-semimodules }} \mathcal{N}^{\bullet}$ with a bounded above complex of projective left $\mathcal{T}_{\text {-semicontramodules }} \mathfrak{F}^{\bullet}$ quasi-isomorphic to $\mathfrak{P}^{\bullet}$.

Here one totalizes the bicomplex $\mathcal{N}^{\bullet} \odot \mathcal{T} \mathfrak{F}^{\bullet}$ by taking infinite direct sums along the diagonals. For any complex of right $\mathcal{T}_{\text {-semimodules }} \mathcal{N}^{\bullet}$, any bounded above complex of left $\mathcal{T}_{\text {-semicontramodules }} \mathfrak{P}^{\bullet}$, and a $k$-vector space $V$ there are natural isomorphisms of $k$-vector spaces

$$
\operatorname{Hom}_{k}\left(\operatorname{CtrTor}_{n}^{\mathcal{T}}\left(\mathcal{N}^{\bullet}, \mathfrak{P}^{\bullet}\right), V\right) \simeq \operatorname{Hom}_{\mathrm{D}(\mathcal{T} \text {-sicntr })}\left(\mathfrak{P}^{\bullet}, \operatorname{Hom}_{k}\left(\mathcal{N}^{\bullet}, V\right)[n]\right) .
$$

Alternatively, the derived functor $\operatorname{CtrTor}^{\mathfrak{T}}$ can be computed using $\mathcal{T} / \mathcal{D}$-projective (or $\mathcal{T} / \mathcal{D}$-contraflat) resolutions of the first argument and $\mathcal{D}$-projective resolutions of the second argument [24, Sections 6.4-5].

A finite complex of right $\mathcal{T}_{\text {-semimodules }} \mathcal{N}^{\bullet}$ is said to have contraflat dimension $\leqslant d$ if one has $\operatorname{Ctr}_{\operatorname{Tor}_{n}^{\mathcal{T}}}^{\mathcal{N}}\left(\mathcal{N}^{\bullet}, \mathfrak{P}\right)=0$ for all left $\mathcal{T}$-semicontramodules $\mathfrak{P}$ and all the integers $n>d$. The contraflat dimension of the complex of right $\mathcal{T}_{\text {-semimodules }}$ $\mathcal{N}^{\bullet}=\mathcal{N}^{\bullet} \square_{\mathcal{D}} \mathcal{T}$ induced from a finite complex of right $\mathcal{D}$-comodules $\mathcal{N} \bullet$ does not exceed the contraflat dimension of the complex of right $\mathcal{D}$-comodules $\mathcal{N}^{\bullet}$. The contraflat dimension of a finite complex of right $\mathcal{T}_{\text {-semimodules }} \mathcal{N}^{\bullet}$ is equal to the injective di-

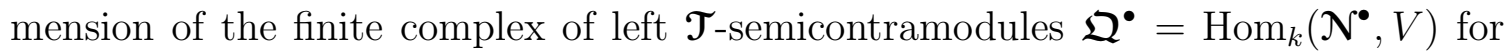
any $k$-vector space $V \neq 0$.

Now we come to the main definition of this section. Let $\mathcal{S}$ be a semialgebra over a coalgebra $\mathcal{C}$ over $k$ and $\mathcal{T}$ be a semialgebra over a coalgebra $\mathcal{D}$ over $k$. Assume that $\mathcal{S}$ is an injective right $\mathcal{C}$-comodule, $\mathcal{T}$ is an injective left $\mathcal{D}$-comodule, the coalgebra $\mathcal{C}$ is left cocoherent, and the coalgebra $\mathcal{D}$ is right cocoherent. A dedualizing complex for $\mathcal{S}$ and $\mathcal{T}$ is defined as a triple consisting of a finite complex of $\mathcal{S}$ - $\mathcal{T}$-bisemimodules $\mathcal{B}^{\bullet}$, a finite complex of $\mathcal{C}$ - $\mathcal{D}$-bicomodules $\mathcal{B}^{\bullet}$, and a morphism of complexes of $\mathcal{C}$ - $\mathcal{D}$-bicomodules $\mathcal{B}^{\bullet} \longrightarrow \mathcal{B}^{\bullet}$ with the following properties:

(iv) $\mathcal{B}^{\bullet}$ is a dedualizing complex for the pair of coalgebras $\mathcal{C}$ and $\mathcal{D}$, that is the conditions (i-iii) of Section 3 are satisfied;

(v) the morphism of complexes of left $\mathcal{S}$-semimodules $\mathcal{S} \square_{\mathcal{C}} \mathcal{B}^{\bullet} \longrightarrow \mathcal{B}^{\bullet}$ induced by the morphism of complexes of left $\mathcal{C}$-comodules $\mathcal{B}^{\bullet} \longrightarrow \mathcal{B}^{\bullet}$ is a quasiisomorphism;

(vi) the morphism of complexes of right $\mathcal{T}_{\text {-semimodules }} \mathcal{B}^{\bullet} \square_{\mathcal{D}} \mathfrak{T} \longrightarrow \mathcal{B}^{\bullet}$ induced by the morphism of complexes of right $\mathcal{D}$-comodules $\mathcal{B}^{\bullet} \longrightarrow \mathcal{B}^{\bullet}$ is a quasiisomorphism.

It follows from the conditions (i) and (v) that the complex $\mathcal{B}^{\bullet}$ has finite projective dimension as a complex of left $\mathcal{S}$-semimodules. Similarly, it follows from the conditions (i) and (vi) that the complex $\mathcal{B}^{\bullet}$ has finite contraflat dimension as a complex of right $\mathcal{T}_{\text {-semimodules. }}$ 
Abusing the terminology, we will sometimes say that the complex of $\mathcal{S}$ - $\mathcal{T}$-bisemimodules $\mathcal{B}^{\bullet}$ is a dedualizing complex (for the semialgebras $\mathcal{S}$ and $\mathcal{T}$ ).

Examples 4.2. Let $\mathcal{S}$ be a semialgebra over a coalgebra $\mathcal{C}$ over $k$ such that the coalgebra $\mathcal{C}$ is left and right cocoherent and left Gorenstein (see Example 3.4), while the semialgebra $\mathcal{S}$ is an injective left $\mathcal{C}$-comodule and an injective right $\mathcal{C}$-comodule. Then the triple consisting of the $\mathcal{S}$-S $\mathcal{S}$-bisemimodule $\mathcal{S}$ (viewed as a one-term complex of $\mathcal{S}$-S -bisemimodules), the $\mathcal{C}$ - $\mathcal{C}$-bicomodule $\mathcal{C}$ (also viewed as a one-term complex of C-C-bicomodules), and the semiunit morphism $\mathcal{C} \longrightarrow \mathcal{S}$ is a dedualizing complex for the pair of semialgebras $(\boldsymbol{S}, \boldsymbol{S})$.

In particular, any semialgebra $\mathcal{S}$ over a cosemisimple coalgebra $\mathcal{C}$ satisfies the above conditions (as cosemisimple coalgebras are co-Noetherian and of homological

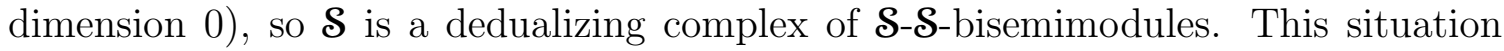
is a rather trivial case for the following theorem, though, as in this case the abelian categories $\mathcal{S}$-simod and $\mathcal{S}$-sicntr are already equivalent (the underived functors $\Psi_{\mathcal{S}}=$ $\operatorname{Hom}_{\mathcal{S}}(\mathcal{S},-)$ and $\Phi_{\mathcal{S}}=\mathcal{S} \odot_{\mathcal{S}}-$ providing the equivalence).

For example, let $G$ be a locally compact totally disconnected (locally profinite) group and $H \subset G$ be a compact open subgroup. Let $k$ be a field. Then the $k$-vector space $\mathcal{C}=k(H)$ of locally constant $k$-valued functions on $H$ has a natural structure of coalgebra over $k$. Moreover, the $k$-vector space $\boldsymbol{S}=k(G)$ of compactly supported locally constant $k$-valued functions on $G$ has a natural structure of semialgebra over $\mathcal{C}$. The semialgebra $\mathcal{S}$ is always an injective left and right $\mathcal{C}$-comodule. The category of (left or right) $\mathcal{S}$-semimodules is isomorphic to the abelian category $G$-smooth sf $_{k}$ of smooth $G$-modules over $k$, while the category of (left or right) $\mathcal{S}$-semicontramodules is isomorphic to the abelian category $G$-contra $k$ of $G$-contramodules over $k$ (see the introduction to [33] and the references therein).

Assume that the proorder of the profinite group $H$ is not divisible by the characteristic of the field $k$. In particular, $G$ can be an arbitrary locally profinite group and $k$ a field of characteristic 0 , or $G$ can be a $p$-adic Lie group, $H \subset G$ an open pro- $p$-subgroup, and $k$ a field of characteristic different from $p$. Then the coalgebra $\mathcal{C}=k(H)$ is cosemisimple. So the semialgebra $\mathcal{S}=k(G)$ is a dedualizing complex of bisemimodules over itself. Moreover, the abelian categories $G$-smooth smd $_{k}$ and $G$-contra ${ }_{k}$ of left $\mathcal{S}$-semimodules and left $\mathcal{S}$-semicontramodules are equivalent.

The situation in the natural characteristic $p$ is more interesting. Let $G$ be a $p$-adic Lie group and $H \subset G$ be a compact open subgroup such that $H$ has no elements of order $p$. Let $k$ be a field of characteristic $p$. Then the coalgebra $\mathcal{C}=k(H)$ is left and right Artinian, since its dual algebra $\mathfrak{C}^{*}=k[[H]]$ is left and right Noetherian. Furthermore, the coalgebra $\mathcal{C}$ has finite homological dimension (equal to the dimension of the group $G$ ). Thus the semialgebra $\mathcal{S}=k(G)$ is a dedualizing complex of $\mathcal{S}$-S-bisemimodules. Hence the following theorem applies (cf. [33]).

Theorem 4.3. Let $\mathcal{S}$ be a semialgebra over a coalgebra $\mathcal{C}$ and $\mathfrak{T}$ be a semialgebra over a coalgebra $\mathcal{D}$ over a field $k$. Assume that the coalgebra $\mathcal{C}$ is left cocoherent, the coalgebra $\mathcal{D}$ is right cocoherent, the semialgebra $\mathcal{S}$ is an injective right $\mathcal{C}$-comodule, 
and the semialgebra $\mathcal{T}$ is an injective left $\mathcal{D}$-comodule. Let $\mathcal{B}^{\bullet} \longrightarrow \mathcal{B}^{\bullet}$ be a dedualizing complex for the semialgebras $\mathcal{S}$ and $\mathcal{T}$. Then for any symbol $\star=\mathrm{b},+,-, \varnothing$, abs+, abs-, or abs there is an equivalence of triangulated categories (8)

$$
\mathrm{D}^{\star}(\mathcal{S} \text {-simod }) \simeq \mathrm{D}^{\star}(\mathcal{T} \text {-sicntr })
$$

provided by mutually inverse functors $\mathbb{R} \operatorname{Hom}_{\mathcal{S}}\left(\mathcal{B}^{\bullet},-\right)$ and $\mathcal{B}^{\bullet} \odot{ }_{\mathcal{T}}^{\mathbb{L}}-$.

Proof. The constructions of the derived functors $\mathbb{R} \operatorname{Hom}_{\mathcal{S}}\left(\mathcal{B}^{\bullet},-\right)$ and $\mathcal{B}^{\bullet} \odot{ }_{\mathcal{T}}^{\mathbb{L}}-$ proceed exactly in the same way as in the proof of Theorem 3.6 (with $\mathcal{C}$ replaced by $\mathcal{S}$, left $\mathcal{C}$-comodules by left $\mathcal{S}$-semimodules, $\mathcal{D}$ replaced by $\mathcal{T}$, left $\mathcal{D}$-contramodules by left $\mathcal{T}_{\text {-semicontramodules, and the complex of bicomodules } \mathcal{B}} \bullet$ replaced by the complex of bisemimodules $\mathcal{B}^{\bullet}$ ). The only property of a finite complex of $\mathcal{S}$ - $\mathcal{T}$-bisemimodules $\mathcal{B}^{\bullet}$ that is used in these constructions is the finiteness of the projective and contraflat dimensions. The result of [29, Appendix B] tells that the two derived functors so obtained are adjoint to each other.

Next it is noticed that the two pairs of adjoint derived functors corresponding to the complex of $\mathcal{C}$ - $\mathcal{D}$-bicomodules $\mathcal{B} \bullet$ and the complex of $\mathcal{S}$ - $\mathcal{T}$-bisemimodules $\mathcal{B}^{\bullet}$ form commutative diagrams with the forgetful functors (cf. the proof of [30, Theorem 5.6])

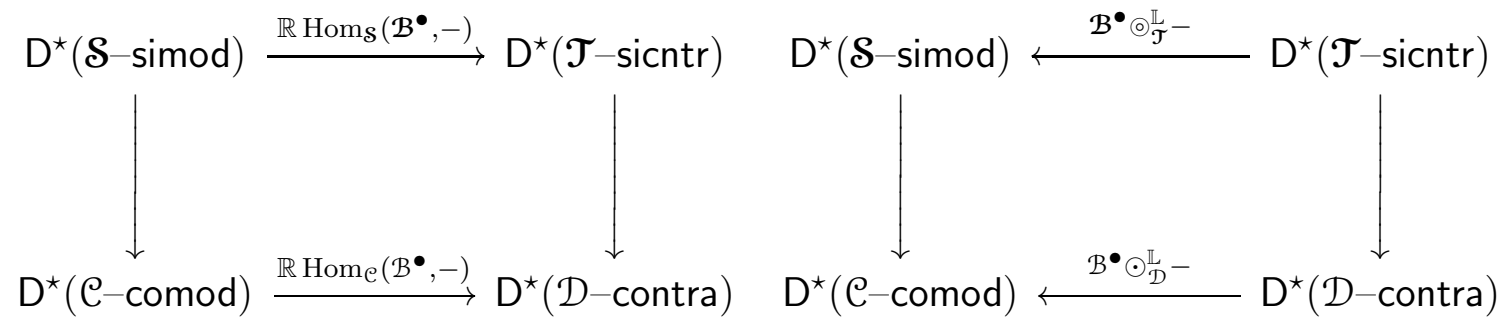

This follows from the conditions (v-vi); the argument is that the total complex of a finite acyclic complex of complexes is absolutely acyclic. The observation that the functor of contratensor product $-\odot_{\mathfrak{T}} \mathfrak{F}$ with a projective left $\mathfrak{T}$-semicontramodule $\mathfrak{F}$ is exact on the abelian category of right $\mathcal{T}$-semimodules simod- $\mathcal{T}$ plays a role here (see the natural isomorphism (9)).

Finally, just as in the proof of Theorem 3.6, checking that the adjunction morphisms for the derived functors $\mathbb{R} \operatorname{Hom}_{\mathcal{S}}\left(\mathcal{B}^{\bullet},-\right)$ and $\mathcal{B}^{\bullet} \odot_{\mathcal{T}}^{\mathbb{L}}-$ are isomorphisms reduces to the case of the bounded derived categories, $\star=\mathrm{b}$. This is a conventional derived category; and for all the conventional derived categories $(\star=b,+,-$, or $\varnothing)$ the forgetful functors $\mathrm{D}^{\star}(\mathcal{S}$-simod $) \longrightarrow \mathrm{D}^{\star}\left(\mathrm{C}_{\text {-comod }}\right)$ and $\mathrm{D}^{\star}(\mathcal{T}$-sicntr $) \longrightarrow \mathrm{D}^{\star}(\mathcal{D}$-contra $)$ are conservative. So it suffices to show that the images of the adjunction morphisms under the forgetful functors are isomorphisms. Similarly to the proof of [30, Theorem 5.6], one observes that these images are nothing but the adjunction morphisms for the derived functors $\mathbb{R} \operatorname{Hom}_{\mathcal{C}}\left(\mathcal{B}^{\bullet},-\right)$ and $\mathcal{B}^{\bullet} \odot_{\mathcal{D}}^{\mathbb{L}}-$. According to the result of Theorem 3.6, we already know that the latter are isomorphisms. 


\section{REFERENCES}

[1] M. Ballard, D. Deliu, D. Favero, M. U. Isik, L. Katzarkov. Resolutions in factorization categories. Advances in Math. 295, p. 195-249, 2016. arXiv:1212.3264 [math.CT]

[2] J. Bernstein, V. Lunts. Equivariant sheaves and functors. Lecture Notes in Math. 1578, Springer-Verlag, Berlin, 1994.

[3] L. W. Christensen, A. Frankild, H. Holm. On Gorenstein projective, injective, and flat dimensions - A functorial description with applications. Journ. of Algebra 302, \#1, p. 231279, 2006. arXiv:math.AC/0403156

[4] W. G. Dwyer, J. P. C. Greenlees. Complete modules and torsion modules. American Journ. of Math. 124, \#1, p. 199-220, 2002.

[5] A. I. Efimov, L. Positselski. Coherent analogues of matrix factorizations and relative singularity categories. Algebra and Number Theory 9, \#5, p. 1159-1292, 2015. arXiv:1102.0261 [math.CT]

[6] J. Gómez-Torrecillas, C. Năstăsescu, B. Torrecillas. Localization in coalgebras. Applications to finiteness conditions. Journ. of Algebra and its Appl. 6, \#2, p. 233-243, 2007. arXiv: math.RA/0403248

[7] J. P. C. Greenlees, J. P. May. Derived functors of $I$-adic completion and local homology. Journ. of Algebra 149, \#2, p. 438-453, 1992.

[8] D. K. Harrison. Infinite abelian groups and homological methods. Annals of Math. 69, \#2, p. 366-391, 1959.

[9] V. Hinich. Homological algebra of homotopy algebras. Communications in Algebra 25, \#10, p. 3291-3323, 1997. arXiv:q-alg/9702015. Erratum, arXiv:math.QA/0309453.

[10] V. Hinich. DG coalgebras as formal stacks. Journ. of Pure and Appl. Algebra 162, \#2-3, p. 209-250, 2001. arXiv:math.AG/9812034

[11] D. Husemoller, J. C. Moore, J. Stasheff. Differential homological algebra and homogeneous spaces. Journ. of Pure and Appl. Algebra 5, \#2, p. 113-185, 1974.

[12] S. Iyengar, H. Krause. Acyclicity versus total acyclicity for complexes over noetherian rings. Documenta Math. 11, p. 207-240, 2006.

[13] P. Jørgensen. The homotopy category of complexes of projective modules. Advances in Math. 193, \#1, p. 223-232, 2005. arXiv:math.RA/0312088

[14] D. Kaledin. Derived Mackey functors. Moscow Math. Journ. 11, \#4, p. 723-803, 2011. arXiv:0812.2519 [math.KT]

[15] B. Keller. Deriving DG-categories. Ann. Sci. de l'École Norm. Sup. (4) 27, \#1, p. 63-102, 1994.

[16] B. Keller. Koszul duality and coderived categories (after K. Lefèvre). October 2003. Available from http://www.math.jussieu.fr/ keller/publ/index.html.

[17] H. Krause. The stable derived category of a Noetherian scheme. Compositio Math. 141, \#5, p. 1128-1162, 2005. arXiv:math.AG/0403526

[18] K. Lefèvre-Hasegawa. Sur les $\mathrm{A}_{\infty}$-catégories. Thèse de doctorat, Université Denis Diderot Paris 7, November 2003. arXiv:math.CT/0310337. Corrections, by B. Keller. Available from

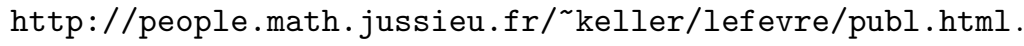

[19] E. Matlis. Cotorsion modules. Memoirs of the American Math. Society 49, 1964.

[20] E. Matlis. The higher properties of $R$-sequences. Journ. of Algebra 50, \#1, p. 77-112, 1978.

[21] J. Miyachi. Derived categories and Morita duality theory. Journ. of Pure and Appl. Algebra 128, \#2, p. 153-170, 1998.

[22] D. Orlov. Matrix factorizations for nonaffine LG-models. Mathematische Annalen 353, \#1, p. 95-108, 2012. arXiv:1101.4051 [math.AG]

[23] M. Porta, L. Shaul, A. Yekutieli. On the homology of completion and torsion. Algebras and Representation Theory 17, \#1, p. 31-67,2014. arXiv:1010.4386 [math.AC] . Erratum in Algebras and Representation Theory 18, \#5, p. 1401-1405, 2015. arXiv:1506.07765 [math.AC] 
[24] L. Positselski. Homological algebra of semimodules and semicontramodules: Semi-infinite homological algebra of associative algebraic structures. Appendix $\mathrm{C}$ in collaboration with D. Rumynin; Appendix D in collaboration with S. Arkhipov. Monografie Matematyczne vol. 70, Birkhäuser/Springer Basel, 2010. xxiv+349 pp. arXiv:0708.3398 [math.CT]

[25] L. Positselski. Two kinds of derived categories, Koszul duality, and comodule-contramodule correspondence. Memoirs of the American Math. Society 212, \#996, 2011. vi+133 pp. arXiv:0905.2621 [math.CT]

[26] L. Positselski. Weakly curved $\mathrm{A}_{\infty}$-algebras over a topological local ring. Electronic preprint arXiv:1202.2697 [math.CT] .

[27] L. Positselski. Contraherent cosheaves. Electronic preprint arXiv:1209.2995 [math.CT] .

[28] L. Positselski. Contramodules. Electronic preprint arXiv:1503.00991 [math.CT] .

[29] L. Positselski. Dedualizing complexes and MGM duality. Journ. of Pure and Appl. Algebra 220, \#12, p. 3866-3909, 2016. arXiv:1503.05523 [math.CT]

[30] L. Positselski. Coherent rings, fp-injective modules, dualizing complexes, and covariant Serre Grothendieck duality. Selecta Math. (New Ser.) 23, \#2, p. 1279-1307, 2017. arXiv: 1504.00700 [math.CT]

[31] L. Positselski. Contraadjusted modules, contramodules, and reduced cotorsion modules. Moscow Math. Journ. 17, \#3, p. 385-455, 2017. arXiv:1605.03934 [math.CT]

[32] L. Positselski. Triangulated Matlis equivalence. Electronic preprint arXiv:1605.08018 [math.CT], to appear in Journ. of Algebra and its Appl.

[33] L. Positselski. Smooth duality and co-contra correspondence. Electronic preprint arXiv: 1609.04597 [math.CT] .

[34] N. Spaltenstein. Resolutions of unbounded complexes. Compositio Math. 65, \#2, p.121-154, 1988.

[35] M. E. Sweedler. Hopf algebras. Mathematics Lecture Note Series, W. A. Benjamin, Inc., New York, 1969.

[36] M. Takeuchi. Morita theorems for categories of comodules. Journ. of the Faculty of Science, the Univ. of Tokyo, Section IA, Math. 24, \#3, p. 629-644, 1977.

[37] M. Wang, Z. Wu. Conoetherian coalgebras. Algebra Colloquium 5, \#1, p. 117-120, 1998.

[38] A. Yekutieli. Dualizing complexes over noncommutative graded algebras. Journ. of Algebra 153, \#1, p. 41-84, 1992.

[39] A. Yekutieli, J. J. Zhang. Rings with Auslander dualizing complexes. Journ. of Algebra 213, \#1, p. 1-51, 1999. arXiv:math.RA/9804005

Department of Mathematics, Faculty of Natural Sciences, University of Haifa, Mount Carmel, Haifa 31905, IsRael; and

Laboratory of Algebraic Geometry, National Research University Higher School OF ECONOMiCS, Moscow 119048; AND

Sector of Algebra and Number Theory, Institute for Information Transmission Problems, Moscow 127051, Russia; And

Charles University in Prague, Faculty of Mathematics and Physics, Department of Algebra, Sokolovská 83, 18675 Praha, Czech Republic

E-mail address: posic@mccme.ru 